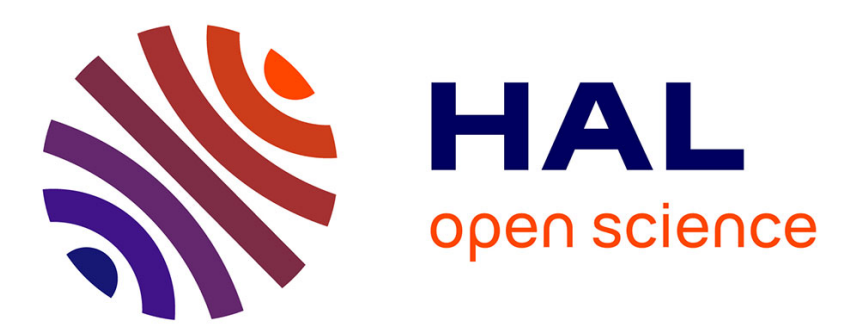

\title{
Articular cartilage with intra- and extrafibrillar waters - Mass transfer and generalized diffusion
}

Benjamin Loret, Fernando M.F. Simões

\section{To cite this version:}

Benjamin Loret, Fernando M.F. Simões. Articular cartilage with intra- and extrafibrillar waters Mass transfer and generalized diffusion. European Journal of Mechanics - A/Solids, 2009, 26 (5), pp.759. 10.1016/j.euromechsol.2007.01.005 . hal-00573074

\section{HAL Id: hal-00573074 \\ https://hal.science/hal-00573074}

Submitted on 3 Mar 2011

HAL is a multi-disciplinary open access archive for the deposit and dissemination of scientific research documents, whether they are published or not. The documents may come from teaching and research institutions in France or abroad, or from public or private research centers.
L'archive ouverte pluridisciplinaire HAL, est destinée au dépôt et à la diffusion de documents scientifiques de niveau recherche, publiés ou non, émanant des établissements d'enseignement et de recherche français ou étrangers, des laboratoires publics ou privés. 


\section{Accepted Manuscript}

Articular cartilage with intra- and extrafibrillar waters - Mass transfer and generalized diffusion

Benjamin Loret, Fernando M.F. Simões

PII: S0997-7538(07)00026-5

DOI: $\quad$ 10.1016/j.euromechsol.2007.01.005

Reference: $\quad$ EJMSOL 2341

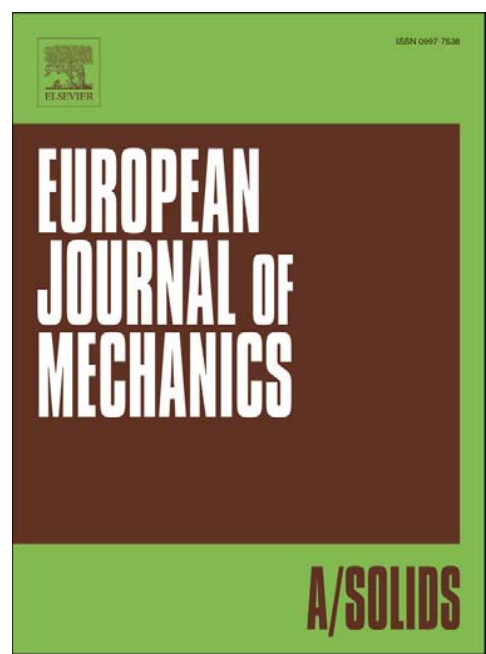

To appear in: $\quad$ European Journal of Mechanics A/Solids

Received date: 6 October 2006

Accepted date: 30 January 2007

Please cite this article as: B. Loret, F.M.F. Simões, Articular cartilage with intra- and extrafibrillar waters - Mass transfer and generalized diffusion, European Journal of Mechanics A/Solids (2007), doi: 10.1016/j.euromechsol.2007.01.005

This is a PDF file of an unedited manuscript that has been accepted for publication. As a service to our customers we are providing this early version of the manuscript. The manuscript will undergo copyediting, typesetting, and review of the resulting proof before it is published in its final form. Please note that during the production process errors may be discovered which could affect the content, and all legal disclaimers that apply to the journal pertain. 


\title{
Articular cartilage \\ with intra- and extrafibrillar waters- \\ Mass transfer and generalized diffusion
}

\author{
Benjamin Loret $^{(1)}$ and Fernando M.F. Simões ${ }^{(2)}$ \\ (1) Laboratoire Sols, Solides, Structures \\ B.P. 53X, 38041 Grenoble Cedex, France \\ (2) ICIST and Departamento de Engenharia Civil, \\ Instituto Superior Técnico, TULisbon \\ Av. Rovisco Pais, 1049-001 Lisboa, Portugal
}

April 2, 2007

\begin{abstract}
The present analysis complements the chemo-mechanical model of articular cartilage developed in Loret and Simões (2004) (2005)a, where only equilibria were considered, and therefore time was absent. The focus here is, first, to present how transport phenomena are aggregated to the porous media framework, and, second, to detail the constitutive equations of these transports. Indeed, these equations are developed in the context of a three-phase multi-species electro-chemo-mechanical model that accounts for the effects of two water compartments, namely intrafibrillar water stored between collagen fibrils and extrafibrillar water covering the negatively charged proteoglycans. The electrolyte circulating the two fluid phases contains ions sodium $\mathrm{Na}^{+}$, calcium $\mathrm{Ca}^{2+}$ and chloride $\mathrm{Cl}^{-}$.

Species diffuse within their phase. They transfer from one fluid phase to the other. The various sources of dissipation are built in a thermodynamic framework, segregated and decoupled via the Clausius-Duhem inequality.

Linear and non linear equations of mass transfer are proposed along an onsagerist approach.

The generalized diffusion in the extrafibrillar compartment accounts for Darcy's law of seepage through the porous solid skeleton, Fick's law of ionic diffusion, and Ohm's law of electric flow. An original derivation of the constitutive equations of generalized diffusion is proposed. Indeed, the dissipation inequality is written in two forms, which are required to be equivalent. This approach has the advantage of delivering the general structure of the diffusion matrix. It also displays in explicit form the degrees of freedom for possible refinements. Simple assumptions, phrased in terms of entities that are standard in transport of porous media, allow to recover arrowhead diffusion matrices. Comparison with an earlier proposal is detailed.

An osmotic coefficient is found to be hidden in the equations, and anomalous negative osmosis is observed to take place for both sodium chloride and calcium chloride electrolytes.

Finally, an experimental setup to measure transport properties is analyzed. The model describes correctly the increase and levelling of the experimental diffusion coefficient, and no additional ad hoc constitutive assumptions are needed in contrast to some suggestions in the literature.

The results are presented for sodium chloride $\mathrm{NaCl}$ and calcium chloride $\mathrm{CaCl}_{2}$.
\end{abstract}

Keywords: cartilage; generalized diffusion; mass transfer; osmotic coefficient 


\section{Introduction}

Articular cartilage is a porous medium, structured by collagen fibers, and saturated by an electrolyte, with water as solvent and metallic ions as solutes. Charged macromolecules, the proteoglycans, intermingled with collagen fibers, give rise to electro-chemo-mechanical couplings that allow moderate deformation to take place, and ensure an optimal adaption of the tissue to physiological loads.

The mechanical significance of the partition of tissue water in intrafibrillar (IF) and extrafibrillar compartments (EF) has been advocated by Maroudas and coworkers. The intrafibrillar compartment is defined as the volume between the collagen fibrils. Proteoglycans (PG's) belong to the extrafibrillar compartment. The presence of ions in the intrafibrillar compartment is constrained by steric considerations : the size of a collagen molecule is $15 \AA$, and the distance between molecules $5 \AA$. Thus water molecules (of size $3.6 \AA$ ), chlorine ions $\mathrm{Cl}^{-}(3.6 \AA)$, sodium cations $\mathrm{Na}^{+}(2 \AA)$, and calcium cations $\mathrm{Ca}^{2+}(2.2 \AA)$ can penetrate the intrafibrillar compartment while larger molecules, like PG's, can not, Torzilli (1985). Experimentally, the partition between intrafibrillar and extrafibrillar water is measured by injecting serum albumin whose size (hydrodynamic size $35 \AA$ ) is large with respect to the intrafibrillar characteristic dimension.

Hydrated PG's induce collagen fibers in tension: a mechanical model would consist of two parallel systems, the pressure induced by PG's being resisted by the applied mechanical load and collagen in tension, Maroudas et al. (1991), Lai et al. (1991), Basser et al. (1998). For unloaded cartilage under physiological salinity, intrafibrillar water represents up to $25 \%$ of total water, the extrafibrillar water furnishing the complement. The latter can be moved by mechanical loading and osmosis with water external to the cartilage (synovial fluid). The intrafibrillar water is in contact with the extrafibrillar compartment only, and it is moved essentially by changes of the chemical composition of the latter. Still, a mechanical loading modifies the relative chemical composition of water (e.g. water being expelled, the concentration of proteoglycans increases), and therefore, indirectly induces a transfer of water from the intrafibrillar to the extrafibrillar compartments.

In Loret and Simões (2004), the two-compartment idea is reconsidered in a hierarchical multi-phase multi-species context. In line with the idea of Maroudas, collagen fibrils behave as a semipermeable membrane, impermeable to macromolecules of molecular mass larger than about $4000 \mathrm{gm}$, and permeable to dissolved metallic ions and water, Li and Katz (1976). They are viewed as separating the two fluid phases.

Experiments of Eisenberg and Grodzinsky (1985) that include both changes of the bath composition and mechanical loads are simulated in Loret and Simões (2004). The latter reference assumes the sole dissolved sodium chloride $\mathrm{NaCl}$ to be present in the cartilage. In Loret and Simões (2005)a, attention is paid to the mechanical interactions between ions, specifically sodium $\mathrm{Na}^{+}$and calcium $\mathrm{Ca}^{2+}$, and the simulations of ionic replacements intertwined with mechanical loadings are performed.

The present work can be viewed as extending to transient and spatially heterogeneous processes the chemo-mechanical model exposed in Loret and Simões (2004)(2005)a which was restricted to equilibria. In other words, the chemical and mechanical loadings were performed with characteristic times much larger than the times characterizing the material response.

The constitutive equations developed here use a thermodynamic framework, that in fact embodies not only purely mechanical aspects, but also transfers of masses between the fluid phases and diffusion of matter through the extrafibrillar phase, Sect. 3. The complete framework 
is used in a companion paper, Loix et al. (2006), to solve, via the finite element method, initial and boundary value problems in view of simulating mechanical and transport phenomena in laboratory specimens submitted to transient mechanical and chemical loading processes. Consequently, the purpose here is, first, to present how the transport phenomena are aggregated to the three-phase context, and second, to detail the constitutive equations of each of these transports.

In fact, several continuum theories have been proposed to model the mechanical and transport behaviors and to describe macroscopic couplings in articular cartilages, Lai et al. (1991), Gu et al. (1998), Huyghe and Janssen (1999). However, their structure is different, as far as the intrafibrillar phase is not recognized. The intra/extrafibrillar split is considered in Huyghe (1999) and Huyghe et al. (2003), but only the mechanical aspects of cartilages bathed in a binary electrolyte are addressed.

While the mechanical behavior is time-independent, the mass transfers and diffusion phenomena addressed here involve typical characteristic times, so that the overall response is time-dependent. Mass transfers relations are directly motivated by the dissipation inequality. Electroneutrality reduces the number of independent transfer relations, which, along an Onsager approach, are proposed in linear and non-linear formats, Sect. 4.

The generalized diffusion in the extrafibrillar compartment accounts for Darcy's law of seepage through the porous solid skeleton, Fick's law of ionic diffusion, Ohm's law of electric flow, electro-osmosis, and the existence of streaming potentials. Strong couplings exist between these phenomena. The internal entropy inequality associated to diffusion is written in two formats: one form involves fluxes relative to the solid, while the second form involves diffusive fluxes relative to the fluid. The equivalence between these two formats is used to structure the diffusion matrix, Sect. 5. The general form of the diffusion equations is provided for a ternary electrolyte, with cations sodium, calcium and anions chloride as mobile ions, Sect. 6.1.

Two simple additional assumptions allow to recover arrowhead diffusion matrices, Sect. 6.2. The present approach has the advantage of displaying in explicit form the complete algebraic structure of the diffusion matrices, and therefore to pave the way for future improvements. Besides, the two above additional assumptions concern entities, that are standard in the modelling of transport in mixtures. Another approach by Gu et al. (1998) consists in working directly on the momentum equations, and in postulating constitutive equations for the momentum transfers between phases: the two methods are mainly equivalent, but the later alternative is more abstract.

The influences of ionic strength and fixed charge density on various key transport properties are discussed, Sect. 6.3. The interactions with mechanical deformation, through the fluid volume fractions, are also underlined.

The tight algebraic structure of the constitutive equations of diffusion implies that the electro-osmotic coefficient is given an explicit form in terms of the fixed charge of the proteoglycans. An osmotic, or reflection, coefficient $\omega$ is also shown to be hidden in the formulation. Therefore, unlike in some other models of charged porous media where the fixed charge is not part of the fluid phase, e.g. Mitchell (1993), Loret et al. (2004), the two above entities, namely the electro-osmotic coefficient and the reflection coefficient, emerge naturally from the formulation, and need not be provided by additional constitutive equations. Two interpretations of the osmotic coefficient are proposed, and, negative osmosis is analyzed in detail and shown to take place for both the sodium chloride and the calcium chloride electrolytes, Sect. 7.

Finally, an experimental setup to measure some transport properties is analyzed, Sect. 8. Data show that the experimental diffusion coefficient increases, from a tiny value, to a plateau as the ionic strength of the bath, the material is in contact with, is increased. This feature is 
shown to be reproduced by the model, and the key effect on the phenomenon of the intensity of the fixed charge is quantified. The data of this setup have been sometimes improperly interpreted, in the geomechanical literature, as providing a basic constitutive relation where the diffusion coefficients are made functions of the transmission coefficient $1-\omega$.

Notation: Vector and tensor quantities are identified by boldface letters. Symbols '.' and ':' between tensors of various orders denote their inner product with single and double contraction respectively. Unless stated otherwise, the convention of summation over repeated indices does not apply.

\section{The framework}

\subsection{Definition of the phases}

The definition of the phases is mechanically motivated. A kinematical criterion on the other hand would classify species according to their velocities. Here, cartilage is viewed as a threephase, multi-species, porous medium, Fig. 1. The solid phase $S$ contains the collagen fibers denoted by the symbol $c$. The intrafibrillar fluid phase $I$ contains intrafibrillar water $w$, ions sodium $\mathrm{Na}^{+}$and calcium $\mathrm{Ca}^{2+}$ and ions chloride $\mathrm{Cl}^{-}$. The extrafibrillar fluid phase $E$ contains proteoglycans, extrafibrillar water, cations sodium and calcium, and anions chloride. The sets of species of the solid, intrafibrillar and extrafibrillar phases are respectively,

$$
S=\{c\}, \quad I=\{w, \mathrm{Na}, \mathrm{Ca}, \mathrm{Cl}\}, \quad E=\{w, \mathrm{PG}, \mathrm{Na}, \mathrm{Ca}, \mathrm{Cl}\} .
$$

A minimum number of cations is required to ensure electroneutrality of the extrafibrillar phase. Therefore, mobile cations are endowed with a velocity independent of that of their non mobile counterparts, which, like the proteoglycans, move with the velocity of the solid phase. Thus cations in the extrafibrillar space are partitioned into a mobile ${ }^{\mathrm{mo}}$ part and a non mobile ${ }^{\mathrm{nm}}$ part. The set $E^{\mathrm{mo}}$ of extrafibrillar mobile species contains the same species as $E$ but proteoglycans. Exchanges of water and ions occur between the fluid phases, but only the extrafibrillar phase communicates with the surroundings, Fig. 1.

The main assumptions, which underly the three-phase multi-species model, follow a strongly interacting model. They have been listed in Loret and Simões (2004) in a purely mechanical perspective. Since deformation, mass transfers and generalized diffusion are accounted for here, they need to be re-stated in this more general context, namely,

(H1) The mass balance is required for each species.

(H2) Momentum balance is required for the mixture as a whole. Water and mobile ions in the extrafibrillar phase are endowed with their own velocities so as to allow the latter to diffuse in their phase and satisfy their own balance of momentum.

(H3) The velocity of any species in the intrafibrillar phase is that of the solid phase, i.e. of collagen, $\mathbf{v}_{k I}=\mathbf{v}_{S}, \forall k \in I$. The velocities of the proteoglycans, and of non mobile cations of the extrafibrillar phase, which do not diffuse through the cartilage are also equal to $\mathbf{v}_{S}$. Thus, the balance of momentum of the above species is not required explicitly, but accounted for by the balance of momentum of the mixture as a whole. Exchange of species between the two fluid phases is viewed as a mass transfer and not as a diffusion process.

(H4) Incompressibility of all the constituents gives rise to a lagrangian pressure $p_{I}$. However, in both the intrafibrillar and extrafibrillar phases, each constituent is endowed, by 
constitutive equations, with its own "generalized pressure", that contains specific contributions in addition to $p_{I}$.

(H5) Electroneutrality is required for the extrafibrillar phase alone, and for the solid and intrafibrillar fluid phases together.

Although the point is seldom mentioned, the definition of phases in articular cartilage is not unambiguous, because the mechanical, chemical and electrical roles of proteoglycans dictate contradictory choices. The consequences of two options are commented further in Remark 7. In particular, proteoglycans move with the solid but their electric charge plays a key role in the extrafibrillar phase. One way to go around this issue is to consider a single (fluid) phase and a single electroneutrality condition, as in Lai et al. (1991). However, topological considerations dictate electroneutrality separately for the collagen fibers and the intrafibrillar content on one hand, and for extrafibrillar water on the other hand. Indeed, when electroneutrality is disturbed, typical times to recover equilibrium are expected to be much smaller than transfer times, so that electroneutrality gets established independently in the two fluid compartments. This aspect might become important when the collagen becomes electrically charged, under non neutral $\mathrm{pH}$.

\subsection{Geometry, mass and work}

Various measures of mass and volume are used to formulate the constitutive equations. They are defined below. Since a species can be present in more than a single phase, it is referred to by two indices, one referring to the species itself, the other to the phase. The only exceptions to this convention are proteoglycans and collagen which unambiguously belong to a single phase.

The current volume (resp. mass) of the species $k$ of phase $K$ is denoted by $V_{k K}$ (resp. $\left.M_{k K}\right)$. Let the initial volume of the porous medium be $V_{0}$ and let $V=V(t)$ be its current volume. Various entities are attached to species:

- some are intrinsic like the intrinsic density $\rho_{k}$, the molar volume $\widehat{v}_{k}$ and molar mass $\widehat{m}_{k}$ linked by $\widehat{m}_{k}=\rho_{k} \widehat{v}_{k}$;

- some refer to the current volume, like the volume fraction $n^{k K}=V_{k K} / V$, and the apparent density $\rho^{k K}=n^{k K} \rho_{k}$;

- some refer to the initial volume like the volume content $v^{k K}=V_{k K} / V_{0}=n^{k K} V / V_{0}$, and the mass content $m^{k K}=M_{k K} / V_{0}=\rho_{k} v^{k K}$.

The associated entities for the phase $K$ are defined by algebraic summation of individual contributions, e.g. the current volume $V_{K}$ and mass $M_{K}$, the volume fraction $n^{K}=V_{K} / V$ (volume fractions satisfy the closure relation $n^{S}+n^{I}+n^{E}=1$ ), the apparent density $\rho^{K}=$ $M_{K} / V$, the volume content $v^{K}=V_{K} / V_{0}=n^{K} V / V_{0}$, and the mass content $m^{K}=M_{K} / V_{0}$.

Other entities live in their phase, e.g. the molar fractions and the concentrations. The molar fraction $x_{k K}$ of the species $k$ in phase $K$ is defined by the ratio of the mole number $N_{k K}$ of that species over the total number of moles within the phase $N_{K}, x_{k K}=N_{k K} / N_{K}$. In each phase, the molar fractions satisfy the closure relation $\sum_{k \in K} x_{k K}=1, K=S, I, E$. Since $N_{k K} / V_{0}=m^{k K} / \widehat{m}_{k}$, the molar fractions can also be expressed in terms of mass contents.

The concentration of an extrafibrillar species is equal to its number of moles referred to the volume of extrafibrillar phase,

$$
c_{k E}^{(\mathrm{mo})}=\frac{N_{k E}^{(\mathrm{mo})}}{V_{E}}=\frac{N_{k E}^{(\mathrm{mo})}}{N_{E} \widehat{v}_{E}}=\frac{1}{\widehat{v}_{k}} \frac{V_{k E}^{(\mathrm{mo})}}{V_{E}}=\frac{1}{\widehat{v}_{k}} \frac{n_{(\mathrm{mo})}^{k E}}{n^{E}}=\frac{1}{\widehat{v}_{k}} \frac{v_{(\mathrm{mo})}^{k E}}{n^{E}} \frac{V_{0}}{V}=\frac{x_{k E}^{(\mathrm{mo})}}{\widehat{v}_{E}}, \quad k \in E,
$$


with $\widehat{v}_{E}=\sum_{k \in E} x_{k E} \widehat{v}_{k}$ the molar volume of the extrafibrillar fluid phase. The superscript (mo) indicates that the definition holds for the entities as a whole, their mobile parts, and their non mobile parts.

Collagen and proteoglycans are macromolecules with a large molar mass, $0.285 \times 10^{6} \mathrm{gm}$ for collagen and $2 \times 10^{6} \mathrm{gm}$ for PG's. The molar fraction $x_{\mathrm{PG}}=N_{\mathrm{PG}} / N_{E}$ and concentration $c_{\mathrm{PG}}=N_{\mathrm{PG}} / V_{E}$ of proteoglycans are thus quite small with respect to the other species of the extrafibrillar phase. On the other hand, the valence $\zeta_{\mathrm{PG}}$ of proteoglycans is large at neutral $\mathrm{pH}$. Thus the effective concentration,

$$
e_{\mathrm{PG}}=\zeta_{\mathrm{PG}} \frac{N_{\mathrm{PG}}}{V_{E}}=\zeta_{\mathrm{PG}} c_{\mathrm{PG}},
$$

is a key parameter of the biochemical and biomechanical behaviors of PG's. For the sake of accuracy in the algebraic derivations, we shall also introduce the notations $\tilde{c}_{\mathrm{PG}}=c_{\mathrm{PG}} n^{E} / n^{w E}$ and $\tilde{e}_{\mathrm{PG}}=e_{\mathrm{PG}} n^{E} / n^{w E}$, even if the ratio $n^{E} / n^{w E}$ is close to one.

Note that the definition of the effective concentration of PG's purposedly refers to the extrafibrillar phase: indeed, according to Maroudas, the effects of PG's is primarily felt in that compartment. However, unlike in the formula (2.3), the effective concentration reported in the literature usually refers to the total volume of the fluid, and, with an opposite sign, it is referred to as fixed charge density (FCD). Maroudas (1975) reports values of FCD between 0.05 and 0.20 mole per liter of total water. According to Gu et al. (1997), the typical value of FCD is about $0.15 \mathrm{M}$ for sound articular cartilage and $0.05 \mathrm{M}$ for osteoarthritic cartilage.

For different zones of the human hip cartilage, Maroudas et al. (1991) indicate values ranging from 0.109 , to 0.210 and 0.228 mole per liter of total water, equivalent to $0.14,0.28$ and 0.32 mole per liter of EF water. Basser et al. (1998) report a mean FCD of 0.37 mole per liter of EF water for normal hip cartilage. This value drops to 0.167 for osteoarthritic cartilage.

While the FCD refers to the amount of extrafibrillar or total water, the cation exchange capacity (CEC) [unit: Coulomb/kg or Coulomb/gm] refers to the dry mass, namely the mass $M_{\text {dry }}$ of collagen and PG's :

$$
\mathrm{CEC}=\mathrm{F}\left|\zeta_{\mathrm{PG}}\right| \frac{N_{\mathrm{PG}}}{M_{\mathrm{dry}}} \Leftrightarrow \mathrm{F} \times \mathrm{FCD}=\mathrm{CEC} \times \frac{n^{\text {dry }}}{n} \rho_{\text {dry }} .
$$

Here $\mathrm{F}=96485$ Coulomb/mole is Faraday's equivalent charge ( 1 Coulomb $=1 \mathrm{~A} \times \mathrm{sec}), n^{\text {dry }}$ the volume fraction of the dry mass, $\rho_{\text {dry }}$ its intrinsic mass density, and $n$ the volume fraction associated to the extrafibrillar fluid or total tissue fluid. In some theories of porous media, this distinction is seen as accounting, or not, for unconnected and dead-end porosities.

For ease of interpretation, Table 1 displays the values of FCD for articular cartilages corresponding to a representative value of CEC, and to representative fluid volume fraction $n$. Note that, during a chemical loading process where the bath composition is varied, the CEC is kept fixed, as far as the proteoglycans are not damaged, while the FCD varies, because the volume fractions vary due to swelling or shrinking.

Table 1. Values of FCD [unit : M] for CEC $=42.9 \mathrm{kC} / \mathrm{kg}, n^{\mathrm{dry}}=1-n, \rho_{\mathrm{dry}}=1800 \mathrm{~kg} / \mathrm{m}^{3}$

\begin{tabular}{c|c|c|c}
\hline \hline$n$ & 0.7 & 0.8 & 0.9 \\
\hline FCD & 0.343 & 0.20 & 0.089
\end{tabular}

The incremental work done by the total stress $\boldsymbol{\sigma}$ in the incremental strain $\delta \boldsymbol{\epsilon}$ of the porous medium and by the electro-chemical potentials $\mu_{k K}^{\mathrm{ec}}$ during the addition of mass $\delta m^{k K}$ of the 
species $k$ to the phase $K$ is

$$
\delta \Psi=\boldsymbol{\sigma}: \delta \boldsymbol{\epsilon}+\sum_{k, K} \mu_{k K}^{\mathrm{ec}} \delta m^{k K}
$$

Here the electro-chemical potentials $\mu_{k K}^{\mathrm{ec}}\left[\right.$ unit: $\mathrm{m}^{2} / \mathrm{sec}^{2}$ ] are mass-based while the fluid-mass contents per unit initial volume of the porous medium $\mathrm{m}^{k K}$ 's are measured in $\mathrm{kg} / \mathrm{m}^{3}$. Note that the above expression assumes infinitesimal strains. An extension to finite strains, while straightforward, e.g. Loret and Simões (2005)b, would not contribute to the issues addressed in this paper.

The chemical potential $\mu_{k K}$ of a species $k$ in phase $K$ identifies a generalized pressure $p_{k K}$, and a chemical contribution which accounts for the molar fraction $x_{k K}$. For a charged species in presence of the electrical potential $\phi^{K}$ [unit Volt $=\mathrm{kg} \times \mathrm{m}^{2} / \mathrm{sec}^{3} / \mathrm{A}$ ], the electro-chemical potential involves in addition an electrical contribution. For incompressible species,

$$
\widehat{m}_{k} \mu_{k K}^{\mathrm{ec}}=\widehat{v}_{k} p_{k K}+R T \operatorname{Ln} x_{k K}+\zeta_{k} \mathrm{~F} \phi^{K}, \quad k \in K .
$$

In this formula, $R=8.31451 \mathrm{~J} / \mathrm{mol} /{ }^{\circ} \mathrm{K}$ is the universal gas constant, $T\left({ }^{\circ} \mathrm{K}\right)$ the absolute temperature. The $\zeta$ 's are the valences.

The extrafibrillar generalized pressures $p_{k E}$ are obtained by constitutive equations: they include in particular a purely mechanical contribution $p_{I}$, a term aimed at satisfaction of chemo-mechanical equilibrium in the hypertonic state, and a term representing the enthalpy of formation, or affinity of ionic species to PG's. The intrafibrillar species are subject to the pressure $p_{I}$. In addition, a hydration mechanism tends to oppose the osmotic flow, induced by PG's, of intrafibrillar water towards the extrafibrillar compartment. The chemo-hyperelastic relations are detailed in Loret and Simões (2004)(2005)a.

\subsection{Electroneutrality}

In phase $K$, the electrical density $I_{\mathrm{e} K}$ [unit: Coulomb $\left./ \mathrm{m}^{3}\right]$ is defined as

$$
I_{\mathrm{e} K}=\frac{\mathrm{F}}{V} \sum_{k \in K} \zeta_{k} N_{k K}\left[=\mathrm{F} n^{E} \sum_{k \in E} \zeta_{k} c_{k E} \text { for } K=E\right] .
$$

For $\mathrm{pH}$ close to 7, the collagen can be considered neutral, Li and Katz (1976). According to Assumption (H5), both the intrafibrillar and extrafibrillar fluid phases are then electrically neutral, $I_{\mathrm{e} I}=I_{\mathrm{e} E}=0$. Electroneutrality requires a minimal number of extrafibrillar cations, that we refer to as non mobile cations: the charge of these cations balances the large negative charge of the proteoglycans, that is

$$
\sum_{k \in E} \zeta_{k} N_{k E}^{\mathrm{nm}}=0, \quad \sum_{k \in E} \zeta_{k} N_{k E}^{\mathrm{mo}}=0
$$

Note however that the non mobile cations sodium do not bind to the PG's. The situation is more complex for cations calcium, as indicated in Sect. 2.5.2.

The electrical potential does not enter the elastic constitutive equations, that can be phrased in terms of chemical potentials, rather than electro-chemical potentials. In fact, the incremental energy (2.5) can be recast in terms of the chemical potentials of water and salts $s_{1}=\mathrm{NaCl}$ and $s_{2}=\mathrm{CaCl}_{2}$ conjugated to the mass contents of water and (mobile) cations sodium and calcium,

$$
\delta \Psi=\boldsymbol{\sigma}: \delta \boldsymbol{\epsilon}+\sum_{K=I, E} \sum_{(i, n) \in\left(K_{\mathrm{in}}, K_{\mathrm{ne}}\right)} \mu_{n K} \delta m^{i K}
$$


with

$$
K_{\mathrm{in}}=\{\mathrm{w}, \mathrm{Na}, \mathrm{Ca}\}, \quad K_{\mathrm{ne}}=\left\{\mathrm{w}, \mathrm{s}_{1}=\mathrm{NaCl}, \mathrm{s}_{2}=\mathrm{CaCl}_{2}\right\} .
$$

Indeed, as a further consequence of electroneutrality, the number of moles of anions chloride is no longer an independent variable and it can be eliminated in favor of the numbers of moles of the cations,

$$
\delta N_{\mathrm{Cl} K}=\sum_{i \in K_{\mathrm{in}}} \zeta_{i} \delta N_{i K}, \quad K=I, E .
$$

The entities $\mu_{n K}, n=s_{1}, s_{2}$, can be viewed as the chemical potentials of the dissociated salts in phase $K$, namely in accordance with (2.6),

$$
\widehat{m}_{i} \mu_{n K}=\left\{\begin{array}{l}
\widehat{m}_{i} \mu_{i K}^{\mathrm{ec}}+\zeta_{i} \widehat{m}_{\mathrm{Cl}} \mu_{\mathrm{Cl} K}^{\mathrm{ec}}, \\
\widehat{v}_{n} p_{n K}+R T \operatorname{Ln} x_{i K}\left(x_{\mathrm{Cl} K}\right)^{\zeta_{i}} .
\end{array}\right.
$$

The molar volumes $\widehat{v}_{n}$ and densities $\rho_{n}$ are defined by the relations,

$$
\widehat{v}_{n}=\rho_{n}^{-1} \widehat{m}_{i}=\widehat{v}_{i}+\zeta_{i} \widehat{v}_{\mathrm{Cl}}, \quad(i, n) \in\left(K_{\mathrm{in}}, K_{\mathrm{ne}}\right) .
$$

The incompressibility of individual species is commonly accepted. Then, the volume change of the whole porous medium $\operatorname{tr} \epsilon$ is equal to the sum of volume changes due to addition/subtraction of individual species, and, Loret and Simões (2005)a,

$$
\delta \operatorname{tr} \boldsymbol{\epsilon}=\sum_{K=I, E} \sum_{(i, n) \in\left(K_{\mathrm{in}}, K_{\mathrm{ne}}\right)} \rho_{n}^{-1} \delta m^{i K} .
$$

\subsection{Balance equations}

The mass balance equations are required for all mobile species in the fluid phases but extrafibrillar water, and for the extrafibrillar fluid phase as a whole. The balances of mass for the intrafibrillar species are accounted for by the transfer relations. The balance of momentum of mobile species of the extrafibrillar phase is accounted for indirectly through the generalized diffusion relations. Balance of momentum for the mixture as a whole is required in a standard format.

\subsubsection{Mass, volume fluxes and electrical current density}

Balance equations are phrased in terms of several fluxes. Because of the incompressibility of the species, mass fluxes and volume fluxes can be viewed as entities that differ only by their units. The mass flux through the solid skeleton $\mathbf{M}_{k K}$ and the associated volume flux $\mathbf{J}_{k K}$ [unit: $\mathrm{m} / \mathrm{s}$ ] of the species $k$ of phase $K$ are defined as,

$$
\rho_{k}^{-1} \mathbf{M}_{k K}=\mathbf{J}_{k K}=n_{\mathrm{mo}}^{k K}\left(\mathbf{v}_{k K}-\mathbf{v}_{S}\right) .
$$

The sum of the fluxes $\mathbf{J}_{k E}, k \in E$, defines the volume averaged flux $\mathbf{J}_{E}$ of the extrafibrillar fluid phase through the solid skeleton,

$$
\mathbf{J}_{E}=\sum_{k \in E} n_{\mathrm{mo}}^{k E}\left(\mathbf{v}_{k E}-\mathbf{v}_{S}\right) .
$$

The PG's do not contribute to the above flux as a consequence of Assumption (H3). 
Let $n_{\mathrm{mo}}^{k E}$ and $n_{\mathrm{nm}}^{k E}$ be a partition of the volume fraction $n^{k E}$ into its mobile and non mobile parts. The diffusive flux with respect to extrafibrillar water is denoted by $\mathbf{J}_{k E}^{d}$,

$$
\mathbf{J}_{k E}^{d}=n_{\mathrm{mo}}^{k E}\left(\mathbf{v}_{k E}-\mathbf{v}_{w E}\right)+n_{\mathrm{nm}}^{k E}\left(\mathbf{v}_{S}-\mathbf{v}_{w E}\right), \quad k \in E .
$$

Relations between the different fluxes are detailed in Appendix A.

The electrical current density $\mathbf{I}_{\mathrm{e} K}$ in phase $K$ [unit: $\left.\mathrm{A} / \mathrm{m}^{2}\right]$ is defined as the sum of constituent velocities weighted by their valences and molar densities,

$$
\mathbf{I}_{\mathrm{e} K}=\mathrm{F} \sum_{k \in K} \zeta_{k} \frac{N_{k K}}{V} \mathbf{v}_{k K}=\mathrm{F} \sum_{k \in K} \zeta_{k} \frac{N_{k K}^{\mathrm{mo}}}{V} \mathbf{v}_{k K} .
$$

The second equality is due to the satisfaction of the electroneutrality by the non mobile ions and PG's. A uniform velocity for all species of a phase satisfying electroneutrality is seen to be a sufficient condition for the electrical current density to vanish in that phase, therefore $\mathbf{I}_{\mathrm{e} I}=\mathbf{0}$. Due to the electroneutrality of both the mobile and non mobile parts in $E, \mathbf{I}_{\mathrm{e} E}$ may be viewed as a sum of either interphase or diffusive fluxes, namely

$$
\mathbf{I}_{\mathrm{e} E}=\mathrm{F} \sum_{k \in E} \zeta_{k} \frac{\mathbf{J}_{k E}}{\widehat{v}_{k}}=\mathrm{F} \sum_{k \in E} \zeta_{k} \frac{\mathbf{J}_{k E}^{d}}{\widehat{v}_{k}} .
$$

\subsubsection{Balances of masses}

The change of mass of a species is due a priori to both transfer, i.e. a physico-chemical reaction, and diffusion. Now, the changes in the species of the intrafibrillar fluid phase are purely reactive, and they are due to transfer, through the membrane that separates the fluid phases, of water and ionic species, Fig. 1. On the other hand, the species of the extrafibrillar fluid phase may also undergo mass changes by exchanges (diffusion) with the surroundings,

$$
\frac{\delta m^{k E}}{\delta t}=-\frac{\delta m^{k I}}{\delta t}-\operatorname{div} \mathbf{M}_{k E}, k \in E .
$$

The symbol div denotes the divergence operator, and $\delta / \delta t$ represents the derivative following the solid phase whose velocity is $\mathbf{v}_{S}$.

For incompressible species, the change of volume of the solid skeleton, which is the same as that of porous medium, is equal and opposite to the change of volume of the extrafibrillar fluid phase due to diffusion,

$$
\operatorname{div} \mathbf{v}_{S}+\operatorname{div} \mathbf{J}_{E}=0 .
$$

As another consequence of (2.20), and of the electroneutrality in the two fluid phases, the electrical current density $\mathbf{I}_{\mathrm{e} E}$ defined by (2.18), or (2.19), may be shown to be divergence free,

$$
\operatorname{div} \mathbf{I}_{\mathrm{e} E}=0 .
$$

\subsubsection{Balance of momentum}

Under quasi-static loading and with the sole gravity $\mathbf{g}$ with intensity $\mathrm{g}$ as body force, the balance of momentum of the porous medium as a whole has the standard format,

$$
\operatorname{div} \boldsymbol{\sigma}+\rho \mathbf{g}=\mathbf{0}
$$

where $\rho$ is the density of the porous medium, i.e. $\rho=\sum_{k, K} \rho^{k K}=\sum_{K} \rho^{K}$. 


\subsection{Calcium partition, screening and binding}

\subsubsection{Partition of mobile and non mobile cations}

The partition between mobile and non mobile cations in the extrafibrillar phase needs to be specified by constitutive relations. If the mobile and non mobile parts of a species were made distinct species belonging to distinct phases, they would be endowed with their own electrochemical potentials, and the interchange between the two species could be viewed as a transfer or chemical reaction, controlled by the differences of their potentials.

However, here, the mechanical effect of a species is considered to be due to the species as a whole, and the chemical potential is defined in terms of the total molar fraction. On the other hand, only the mobile part of the species can diffuse through the extrafibrillar water.

If there is a single cation $k$ present in the solution, its partition in mobile and non mobile parts is known, namely its non mobile part is defined by the relation $\zeta_{k} N_{k E}^{\mathrm{nm}}+\zeta_{\mathrm{PG}} N_{\mathrm{PG}}=0$. When the two cations sodium and calcium are present, their partition needs to be defined. One might tentatively endow the mobile and non mobile parts with chemical potentials and the partition will be defined by the equilibrium constant $K_{\text {eq }}$ that differentiates the affinities of cations sodium and calcium for proteoglycans, namely in terms of concentrations

$$
\frac{\left(c_{\mathrm{Na} E}^{\mathrm{nm}}\right)^{2}}{\left(c_{\mathrm{Na} E}^{\mathrm{mo}}\right)^{2}}=K_{\mathrm{eq}} \frac{c_{\mathrm{Ca} E}^{\mathrm{nm}}}{c_{\mathrm{Ca} E}^{\mathrm{mo}}} .
$$

Therefore the concentrations can be obtained from the single positive solution of the equation

$$
\left(c_{\mathrm{Na} E}^{\mathrm{nm}}\right)^{2} \overbrace{\frac{c_{\mathrm{Cl} E}-c_{\mathrm{Na} E}^{\mathrm{mo}}}{K_{\mathrm{eq}}\left(c_{\mathrm{Na} E}^{\mathrm{mo}}\right)^{2}}}^{\geq 0}+c_{\mathrm{Na} E}^{\mathrm{nm}}+\overbrace{e_{\mathrm{PG}}}^{<0}=0 .
$$

The above discussion assumes that the extrafibrillar $\mathrm{pH}$ is above the isoelectric point, in which case the electrical charge of PG's is indeed negative.

\subsubsection{Calcium binding to the fixed charge}

The fixed charge of polyelectrolyte gels of biological interest and of active clays are known to be $\mathrm{pH}$-sensitive. Indeed, as the $\mathrm{pH}$ of the surrounding decreases (resp. increases), the fixed charge becomes less negative (resp. more negative). For active clays, the issue is considered on both experimental and modeling points of view in Gajo and Loret (2007). Indeed, the change of charge is due to surface complexation mechanisms involving cations hydrogen $\mathrm{H}^{+}$and anions hydroxyl $\mathrm{OH}^{-}$.

The change of fixed charge might be due to the binding of other ions. Cations calcium are known to bind, at least partially, to proteoglycans, while cations sodium do not. At sufficiently high calcium concentration, the binding might become irreversible and modify the structure of the proteoglycans. The ability of proteoglycans to bind calcium has been advocated to contribute to the calcification process of articular cartilage, Werner and Gründer (1999).

Still the phenomenon remains to be quantified. Moreover it is known to be very sensitive to a number of histological details, for example chondroitin sulfate 4 is more prone to bind calcium than chondroitin sulfate 6 .

However, the present analysis considers that the fixed charge is permanent, and cation binding is not accounted for. In other words, a distinction among the non mobile calcium cations into free and binded cations is not performed. 


\section{The global structure: deformation, mass transfer, diffusion}

The constitutive equations are developed in a thermodynamic framework à la Biot where the solid skeleton is taken as reference. Such an approach has been derived in a more general context including in addition the growth phenomenon in Loret and Simões (2005)b. A single inequality for the internal entropy is required for the porous medium as a whole. It results in an expression that contains three terms of distinct natures, and which consequently are required to be positive individually,

$$
\left\{\begin{array}{l}
\delta D_{1}=-\delta \Psi+\boldsymbol{\sigma}: \delta \boldsymbol{\epsilon}+\sum_{k, K} \mu_{k K} \delta m^{k K} \geq 0 \\
\delta D_{2}=-\sum_{k \in I}\left(\mu_{k I}-\mu_{k E}\right) \delta m^{k I} \geq 0 \\
\delta D_{3} / \delta t=-\sum_{k \in E^{\mathrm{mo}}}\left(\nabla \mu_{k E}^{\mathrm{ec}}-\mathbf{b}_{k E}\right) \cdot \mathbf{M}_{k E} \geq 0
\end{array}\right.
$$

The chemo-hyperelastic behavior is constructed in order for the first term $\delta D_{1}$ to exactly vanish, Loret and Simões (2004)(2005)a. Due to phase electroneutrality, the electrical field does not work, and this has lead to the electro-chemical potentials in $\delta D_{1}$ and in $\delta D_{2}$ to be replaced by the chemical potentials. Consequently, the elastic relations and the transfer relations do not depend directly on the electrical field, as already mentioned in a different format in Section 2.3.

Satisfaction of the second and third inequalities motivates generalized transfer equations and generalized diffusion equations respectively: they are developed in the subsequent sections. For quasi-static analyses, the body force densities $\mathbf{b}_{k E}$ are equal to the gravity $\mathbf{g}$, and individual accelerations are neglected. Note that uniform body forces can be viewed as introducing a sedimentation contribution into the electro-chemo-mechanical potentials.

\section{Mass transfers as physico-chemical reactions}

In view of the electroneutrality of the fluid phases, the inequality dissipation associated to mass transfer can be recast in terms of the independent intrafibrillar variables $I_{\text {in }}$, namely

$$
\delta D_{2}=-\sum_{(i, n) \in\left(I_{\mathrm{in}}, I_{\mathrm{ne}}\right)}\left(\mu_{n I}-\mu_{n E}\right) \delta m^{i I} \geq 0
$$

This inequality can be recast in vector form, $\delta D_{2}=-\mathbf{X} \cdot \delta \mathbf{Y} \geq 0$, with

$$
\mathbf{Y}=\left[\begin{array}{c}
m^{w I} \\
m^{\mathrm{Na} I} \\
m^{\mathrm{Ca} I}
\end{array}\right], \quad \mathbf{X}=\left[\begin{array}{c}
\mu_{w I}-\mu_{w E} \\
\mu_{s_{1} I}-\mu_{s_{1} E} \\
\mu_{s_{2} I}-\mu_{s_{2} E}
\end{array}\right]
$$

The simplest way to satisfy the dissipation inequality is to introduce the linear transfer relations,

$$
\frac{\delta \mathbf{Y}}{\delta t}=-\mathbf{T} \cdot \mathbf{X}
$$

Dissipation is ensured if the $n_{\text {ion }} \times n_{\text {ion }}$ transfer matrix $\mathbf{T}$ is symmetric positive (semi-)definite. As a simplification, uncoupling between the mass transfers amounts to a diagonal transfer matrix. It implies that the sole chemical out-of-equilibrium of water does not result in cation transfers, and conversely. Each of the transfer equations is then characterized by a characteristic transfer time $\tau_{i}, i \in I_{\text {in }}$, and a configuration constant $K_{\mathrm{c} n}, n \in I_{\mathrm{ne}}$, used to define the equilibrium 
constant. The transfer times can be displayed by dimension analysis of the diagonal terms of the matrix $\mathbf{T}$,

$$
T_{i}=A_{i} \frac{\rho_{i}}{\tau_{i}}, i \in I_{\mathrm{in}}
$$

where $A_{i}$ [unit: $\sec ^{2} / \mathrm{m}^{2}$ ] is a material parameter.

More general transfer rules that satisfy the dissipation inequality can be postulated if the above uncoupling holds. Indeed, consider the inequality $-\mu d m / d t \geq 0$, where $\mu$ is understood as the difference $\mu_{w I}-\mu_{w E}$. The linear rate equation $d m / d t=-A \mu$ satisfies the inequality if $A$ is a positive scalar. So does the rate equation $d m / d t=(\exp (-\epsilon A \mu)-1) \epsilon$, with $\epsilon= \pm 1$. While the latter rate equation reduces to the linear equation at small $\mu$, its behavior at large positive and negative $\mu$ depends strongly on $\epsilon$, Fig. 2 .

Consider now the transfer of water with $\epsilon=1$. If the intrafibrillar pressure of water is large, then the above $\mu$ is positive, water tends to leave the intrafibrillar compartment, but the trend to exhaustion of the intrafibrillar water is limited. On the other hand, if the intrafibrillar pressure is much smaller than the extrafibrillar pressure, the rate of replenishment becomes exponential. The value $\epsilon=-1$ has converse effects on the rate of in- and outflow of water, Fig. 2. The same reasoning applies for the transfer of ions.

As a final remark, observe that the rate relation

$$
\frac{\delta m_{i I}}{\delta t}=\frac{\rho_{i}}{\tau_{i}}\left[\exp \left(-A_{i}\left|\mu_{n I}-\mu_{n E}\right|\right)-1\right] s_{\mu}, \quad(i, n) \in\left(I_{\mathrm{in}}, I_{\mathrm{ne}}\right)
$$

with $s_{\mu}=\operatorname{sign}\left(\mu_{n I}-\mu_{n E}\right)$, is an example of transfer law that 1 . is motivated by, and satisfies the inequality dissipation, 2. ensures a finite rate of transfer at any concentration, and 3. reduces to the linear transfer law (4.3) close to equilibrium.

\section{Generalized diffusion: formal constitutive equations}

Inequality $\delta D_{3} \geq 0$, eqn (3.1), is ensured by generalization of Darcy's law of seepage through the porous medium, Fick's law of diffusion of ions in the extrafibrillar fluid phase, and Ohm's law of electrical flow. There are at least two ways of using the inequality to build the generalized diffusion equations. However, the end results of the two separate developments are shown below to be equivalent, and the relations between the coefficients of the two generalized diffusion matrices introduced, of respective sizes $\left(1+n_{\text {ion }}\right) \times\left(1+n_{\text {ion }}\right)$ and $\left(2+n_{\text {ion }}\right) \times\left(2+n_{\text {ion }}\right)$, are provided. $n_{\text {ion }}$ is the number of mobile ionic species, namely three for a ternary electrolyte.

An electro-osmotic coefficient and an osmotic, or reflection, coefficient are shown to emerge naturally from the formulation.

The analysis assumes the constitutive equations for generalized diffusion to be isotropic. Extension to account for anisotropy in Darcy's law, Fick's law and Ohm's law is formally straightforward, but, to be useful, would require data to be available.

\subsection{Two equivalent forms of the dissipation due to generalized diffusion}

\subsubsection{Diffusion in terms of fluxes relative to the solid}

An immediate way to satisfy the inequality $\delta D_{3} \geq 0$, eqn (3.1), is to postulate the existence of a symmetric positive (semi-)definite matrix (PsD) $\boldsymbol{\kappa}$ that provides the volume fluxes as a function of the electro-chemical potentials, namely,

$$
\mathbf{j}=-\boldsymbol{\kappa} \mathbf{f}, \boldsymbol{\kappa}=\boldsymbol{\kappa}^{T} \mathrm{PSD},
$$


that is, formally,

$$
\mathbf{j}=\left[\begin{array}{c}
\mathbf{J}_{w E} \\
\mathbf{J}_{\mathrm{Na} E} \\
\mathbf{J}_{\mathrm{Ca} E} \\
\mathbf{J}_{\mathrm{Cl} E}
\end{array}\right], \quad \mathbf{f}=\left[\begin{array}{c}
\rho_{w} \nabla \mu_{w E} \\
\rho_{\mathrm{Na}} \nabla \mu_{\mathrm{Na} E}^{\mathrm{ec}} \\
\rho_{\mathrm{Ca}} \nabla \mu_{\mathrm{Ca} E}^{\mathrm{ec}} \\
\rho_{\mathrm{Cl}} \nabla \mu_{\mathrm{Cl} E}^{\mathrm{ec}}
\end{array}\right], \quad \boldsymbol{\kappa}=\left[\begin{array}{cccc}
\kappa_{w w} & \kappa_{w \mathrm{Na}} & \kappa_{w \mathrm{Ca}} & \kappa_{w \mathrm{Cl}} \\
\kappa_{\mathrm{Na} w} & \kappa_{\mathrm{NaNa}} & \kappa_{\mathrm{NaCa}} & \kappa_{\mathrm{NaCl}} \\
\kappa_{\mathrm{Ca} w} & \kappa_{\mathrm{CaNa}} & \kappa_{\mathrm{CaCa}} & \kappa_{\mathrm{CaCl}} \\
\kappa_{\mathrm{Cl} w} & \kappa_{\mathrm{ClNa}} & \kappa_{\mathrm{Cl} \mathrm{a}} & \kappa_{\mathrm{ClCl}}
\end{array}\right] .
$$

In fact the choice of the conjugate pair of fluxes and forces contains two degrees of arbitrariness. Indeed, let $\alpha$ be an arbitrary strictly positive scalar, and $\boldsymbol{\Delta}$ be an arbitrary, but invertible, diagonal matrix. Then the pair $(\overline{\mathbf{j}}, \overline{\mathbf{f}})$, defined by

$$
\overline{\mathbf{j}}=\alpha \boldsymbol{\Delta} \mathbf{j}, \quad \overline{\mathbf{f}}=\boldsymbol{\Delta}^{-1} \mathbf{f},
$$

satisfies

$$
\delta D_{3} / \delta t=-\mathbf{f}^{T} \mathbf{j}=-\alpha^{-1} \overline{\mathbf{f}}^{T} \overline{\mathbf{j}} \geq 0 .
$$

Therefore it is equivalent to postulate the generalized diffusion law on the pairs $(\mathbf{j}, \mathbf{f})$ and $(\overline{\mathbf{j}}, \overline{\mathbf{f}})$. The symmetry and positive definiteness of the diffusion matrix $\boldsymbol{\kappa}$ carry over to the diffusion matrix $\bar{\kappa}$, and conversely,

$$
\overline{\mathbf{j}}=-\overline{\boldsymbol{\kappa}} \overline{\mathbf{f}}, \quad \overline{\boldsymbol{\kappa}}=\alpha \Delta \boldsymbol{\kappa} \boldsymbol{\Delta} .
$$

\subsubsection{Diffusion in terms of diffusive fluxes and current density}

A more familiar form, where the diffusive fluxes and electric current density appear, emerges by using the expression of the electro-chemical potential (2.6), the closure relation satisfied by the molar fractions, and eqns (2.15),(2.17). Then the dissipation inequality $\delta D_{3} \geq 0$ is obtained as the sum of four terms:

- the pressure term can be simplified as follows. First, the gradients of the generalized pressures $\nabla p_{k E}$ are all identical in the model developed in Loret and Simões (2004)(2005)a. Second, the total flux $\mathbf{J}_{E}$ may be approximated by the flux of water $\mathbf{J}_{w E}$. Then

$$
\sum_{k \in E^{\mathrm{mo}}} \nabla p_{k E} \cdot \mathbf{J}_{k E} \simeq \nabla p_{w E} \cdot \mathbf{J}_{w E}
$$

- the chemical term can be additively decomposed, using (A.1), in the classic term plus a term due to the presence of $\mathrm{PG}$ in the extrafibrillar phase,

$$
\sum_{k \in E^{\mathrm{mo}}} R T \nabla \operatorname{Ln} x_{k E} \cdot \frac{\mathbf{J}_{k E}}{\widehat{v}_{k}}=\sum_{k \in E^{\mathrm{ions}}} R T \nabla \operatorname{Ln} x_{k E} \cdot \frac{\mathbf{J}_{k E}^{d}}{\widehat{v}_{k}}-R T \nabla \tilde{c}_{\mathrm{PG}} \cdot \mathbf{J}_{w E}
$$

- the electrical term is simply

$$
\nabla \phi_{E} \cdot \mathbf{I}_{\mathrm{e} E}
$$

- in the gravity term, using (A.6),

$$
\sum_{k \in E^{\mathrm{mo}}}-\rho_{k} \mathbf{g} \cdot \mathbf{J}_{k E}=-\mathbf{g} \cdot\left(\frac{\rho_{*}^{E}}{n_{*}^{E}} \mathbf{J}_{E}+\sum_{l \in E^{\mathrm{mo}}}\left(\rho_{k}-\frac{\rho_{*}^{E}}{n_{*}^{E}}\right) \mathbf{J}_{k E}^{d}\right),
$$

the total flux $\mathbf{J}_{E}$ may be approximated once again by the flux of water $\mathbf{J}_{w E}$. 
To derive the above expressions, repeated use has been made of the relation: $\nabla \operatorname{Ln} x_{w E} / \widehat{v}_{w}=$ $-\nabla \tilde{c}_{\mathrm{PG}}-\sum_{l \in E^{\text {ions }}} n^{l E} / n^{w E} \nabla \operatorname{Ln} x_{l E} / \widehat{v}_{l}$.

Therefore, equivalent to $(3.1)_{3}$, the dissipation inequality can be formally written as the sum of products of a flux times a driving force,

$$
\delta D_{3} / \delta t=-\mathcal{F}^{T} \mathcal{J}=-\mathbf{F}_{E} \cdot \mathbf{J}_{w E}-\sum_{k \in E^{\mathrm{ions}}} \mathbf{F}_{k E}^{d} \cdot \mathbf{J}_{k E}^{d}-\mathbf{F}_{\mathrm{e} E} \cdot \mathbf{I}_{\mathrm{e} E} \geq 0
$$

The vector flux $\mathcal{J}$ and its conjugate vector $\mathcal{F}$ have now $2+n_{\text {ion }}$ entries,

$$
\mathcal{J}=\left[\begin{array}{c}
\mathbf{J}_{w E} \\
\mathbf{J}_{\mathrm{Na} E}^{d} \\
\mathbf{J}_{\mathrm{Ca} E}^{d} \\
\mathbf{J}_{\mathrm{Cl} E}^{d} \\
\mathbf{I}_{\mathrm{e} E}
\end{array}\right], \quad \mathcal{F}=\left[\begin{array}{c}
\mathbf{F}_{E} \\
\mathbf{F}_{\mathrm{Na} E}^{d} \\
\mathbf{F}_{\mathrm{Ca} E}^{d} \\
\mathbf{F}_{\mathrm{Cl} E}^{d} \\
\mathbf{F}_{\mathrm{e} E}
\end{array}\right]=\left[\begin{array}{l}
\nabla P_{w E}-\rho_{*}^{E} / n_{*}^{E} \mathbf{g} \\
R T / \widehat{v}_{\mathrm{Na}} \nabla \operatorname{Ln} x_{\mathrm{Na} E}-\left(\rho_{\mathrm{Na}}-\rho_{*}^{E} / n_{*}^{E}\right) \mathbf{g} \\
R T / \widehat{v}_{\mathrm{Ca}} \nabla \operatorname{Ln} x_{\mathrm{Ca} E}-\left(\rho_{\mathrm{Ca}}-\rho_{*}^{E} / n_{*}^{E}\right) \mathbf{g} \\
R T / \widehat{v}_{\mathrm{Cl}} \nabla \operatorname{Ln} x_{\mathrm{Cl} E}-\left(\rho_{\mathrm{Cl}}-\rho_{*}^{E} / n_{*}^{E}\right) \mathbf{g} \\
\nabla \phi_{E}
\end{array}\right],
$$

where $\nabla P_{w E}=\nabla p_{w E}-R T \nabla \tilde{c}_{\mathrm{PG}}$.

The generalized diffusion law can be expressed via a symmetric matrix $\mathcal{K}$ of size $\left(2+n_{\text {ion }}\right) \times$ $\left(2+n_{\text {ion }}\right)$,

$$
\mathcal{J}=-\mathcal{K} \mathcal{F}, \quad \mathcal{K}=\mathcal{K}^{T} \operatorname{PsD}
$$

with components

$$
\left[\begin{array}{ccccc}
k_{E E} & k_{E \mathrm{Na}}^{d} & k_{E \mathrm{Ca}}^{d} & k_{E \mathrm{Cl}}^{d} & k_{\mathrm{e}} \\
k_{\mathrm{Na} E}^{d} & k_{\mathrm{NaNa}}^{d} & k_{\mathrm{NaCa}}^{d} & k_{\mathrm{NaCl}}^{d} & k_{\mathrm{Nae}}^{d} \\
k_{\mathrm{Ca} E}^{d} & k_{\mathrm{CaNa}}^{d} & k_{\mathrm{CaCa}}^{d} & k_{\mathrm{CaCl}}^{d} & k_{\mathrm{Cae}}^{d} \\
k_{\mathrm{Cl} E}^{d} & k_{\mathrm{ClNa}}^{d} & k_{\mathrm{ClCa}}^{d} & k_{\mathrm{ClCl}}^{d} & k_{\mathrm{Cle}}^{d} \\
k_{\mathrm{e}} & k_{\mathrm{eNa}}^{d} & k_{\mathrm{eCa}}^{d} & k_{\mathrm{eCl}}^{d} & \sigma_{\mathrm{e}}
\end{array}\right]
$$

Remark 1: On the dependence of the components of $\mathcal{J}$ and $\mathcal{F}$

The components $\mathbf{F}_{\mathrm{Na} E}^{d}, \mathbf{F}_{\mathrm{Ca} E}^{d}$ and $\mathbf{F}_{\mathrm{Cl} E}^{d}$ of the vector $\mathcal{F}$ are linearly independent, even in absence of gravity, due to the presence of PG's. In fact, extrafibrillar electroneutrality implies

$$
\sum_{k \in E^{\text {ions }}} \zeta_{k} c_{k E} \widehat{v}_{k} \mathbf{F}_{k E}^{d}=-R T \nabla e_{\mathrm{PG}}
$$

On the other hand, the components of the flux $\mathcal{J}$ are linearly dependent. Indeed the electrical current density $\mathbf{I}_{\mathrm{e} E}$ is a linear combination of the ionic diffusive fluxes $\mathbf{J}_{k E}^{d}$, eqn $(2.19)_{2}$, and of the flux of water, which is proportional to the diffusive flux of PG's,

$$
\mathbf{I}_{\mathrm{e} E}-\mathrm{F} \sum_{k \in E^{\text {ions }}} \zeta_{k} \frac{\mathbf{J}_{k E}^{d}}{\widehat{v}_{k}}=\mathrm{F} \zeta_{\mathrm{PG}} \frac{\mathbf{J}_{\mathrm{PG}}^{d}}{\widehat{v}_{\mathrm{PG}}}=-\mathrm{F} \tilde{e}_{\mathrm{PG}} \mathbf{J}_{w E} .
$$

\section{Remark 2: Positive definiteness of the diffusion matrix $\mathcal{K}$}

As a consequence of the above remark, the lines of the diffusion matrix $\mathcal{K}$ are linearly dependent and $\mathcal{K}$ can be at best positive semi-definite (PsD). That the matrices $\boldsymbol{\kappa}$ and $\mathcal{K}$ are 
actually PsD will be addressed in Remark 4 below.

\section{Remark 3: Identification procedures}

Any identification of the diffusion coefficients uses data from specific experimental processes. There are typically two main methods. The generalized diffusion coefficients can be obtained from ionic mobilities measured in experiments

- either at vanishing pore pressure gradient $\mathbf{F}_{E}=\mathbf{0}$, in which case the diffusive fluxes simplify to

$$
\mathbf{J}_{k E}^{d}=-\sum_{l \in E^{\text {ions }}} k_{k l}^{d} \mathbf{F}_{l E}^{d}-k_{k \mathrm{e}}^{d} \mathbf{F}_{\mathrm{e} E}, \quad k \in E^{\mathrm{ions}},
$$

- or at vanishing water flux $\mathbf{J}_{w E}=\mathbf{0}$, and then

$$
\mathbf{J}_{k E}^{d}=-\sum_{l \in E^{\text {ions }}}\left(k_{k l}^{d}-\frac{k_{E k}^{d} k_{l E}^{d}}{k_{E E}}\right) \mathbf{F}_{l E}^{d}-\left(k_{k \mathrm{e}}^{d}-\frac{k_{\mathrm{e}}}{k_{E E}} k_{E k}^{d}\right) \mathbf{F}_{\mathrm{e} E}, \quad k \in E^{\mathrm{ions}} .
$$

\subsection{Relations between the $4 \times 4$ and $5 \times 5$ diffusion matrices}

As noted above, the simplest way to define the diffusion properties would be to identify directly the matrix $\boldsymbol{\kappa}$. Its coefficients are a priori independent and they are restricted only by symmetry and positive (semi-)definiteness. However, more information is available on the matrix $\mathcal{K}$. But this matrix $\mathcal{K}$ is not definite, and therefore there exist relations between its coefficients.

So there are two ways of proceeding:

- either postulate directly the matrix $\mathcal{K}$ and unveil the compatibility between its coefficients due to (5.15) and symmetry, as done in Loret et al. (2004) in their analysis of two-phase clays where the fixed charges (clay platelets) belong to the solid phase. Note that the fact that the fixed charge belong or not to the extrafibrillar compartment has far reaching consequences, as stressed in Remark 7;

- or use directly the compatibility relations inferred by the relations between the coefficients of the matrices $\kappa$ and $\mathcal{K}$. This is the method followed here. As a by-product of the identification, the coefficients of the matrix $\boldsymbol{\kappa}$ will be known.

It is instrumental to introduce the coefficients,

$$
\kappa_{k l}^{d} \equiv \kappa_{k l}-\frac{n^{k E}}{n^{w E}} \kappa_{w l}, \quad k \in E^{\mathrm{ions}}, l \in E^{\mathrm{mo}} .
$$

The coefficients of the matrix $\mathcal{K}$ are given in terms of those of the matrix $\boldsymbol{\kappa}$ as follows:

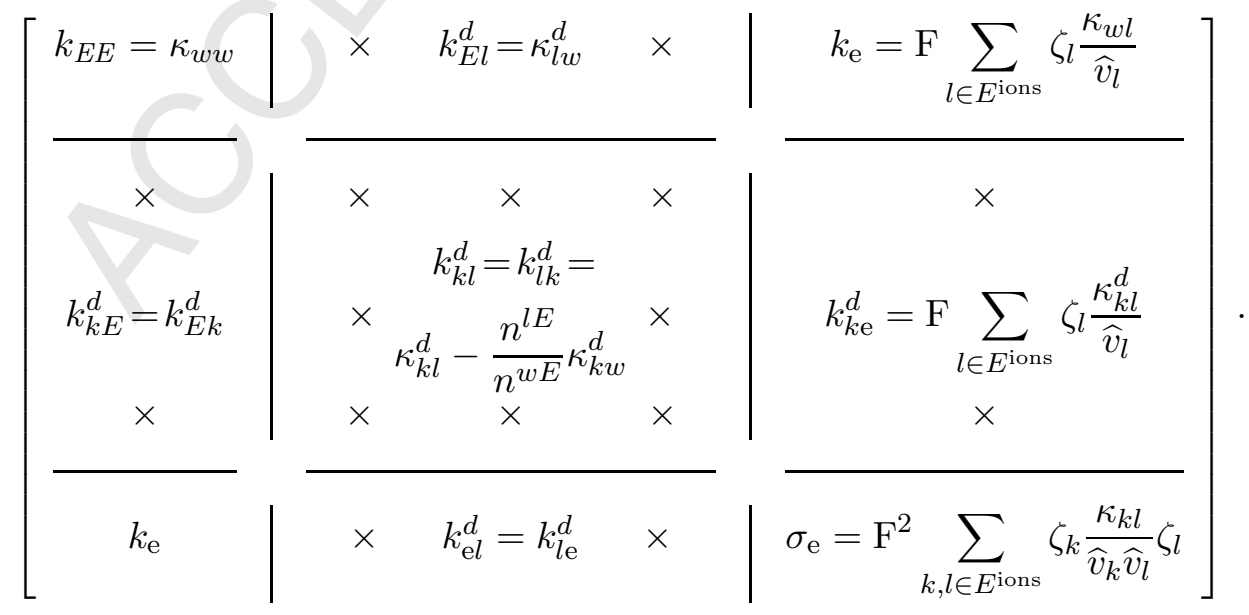


The symmetry of the matrix $\boldsymbol{\kappa}$ has been used to establish (5.19). The matrix $\mathcal{K}$ inherits the symmetry property: even if a priori $\kappa_{k l}^{d} \neq \kappa_{l k}^{d}$, for $k \neq l$, the central coefficients do so, in fact

$$
k_{k l}^{d}=k_{l k}^{d}=\kappa_{k l}-\frac{n^{k E}}{n^{w E}} \kappa_{l w}-\frac{n^{l E}}{n^{w E}} \kappa_{w k}+\frac{n^{k E}}{n^{w E}} \frac{n^{l E}}{n^{w E}} \kappa_{w w}, \quad k, l \in E^{\mathrm{ions}} .
$$

The $2+n_{\text {ion }}$ compatibility relations that the coefficients of the generalized diffusion matrix $\mathcal{K}$ have to satisfy due to the relation of linear dependence (5.15) can now be cast in the format

$$
\left\{\begin{array}{l}
k_{\mathrm{e}}=\mathrm{F} \sum_{l \in E^{\mathrm{ions}}} \zeta_{l} \frac{k_{l E}^{d}}{\widehat{v}_{l}}-\mathrm{F} \tilde{e}_{\mathrm{PG}} k_{E E}, \\
k_{\mathrm{e} k}^{d}=\mathrm{F} \sum_{l \in E^{\text {ions }}} \zeta_{l} \frac{k_{k l}^{d}}{\widehat{v}_{l}}-\mathrm{F} \tilde{e}_{\mathrm{PG}} k_{k E}^{d}, \quad k \in E^{\mathrm{ions}} \\
\sigma_{\mathrm{e}}=\mathrm{F} \sum_{l \in E^{\text {ions }}} \zeta_{l} \frac{k_{l \mathrm{e}}^{d}}{\widehat{v}_{l}}-\mathrm{F} \tilde{e}_{\mathrm{PG}} k_{\mathrm{e}} .
\end{array}\right.
$$

A particular consequence of these compatibility relations is worth to be recorded, namely

$$
\mathrm{F}^{2} \sum_{k, l \in E^{\text {ions }}} \zeta_{k} \frac{k_{k l}^{d}}{\widehat{v}_{k} \widehat{v}_{l}} \zeta_{l}=\sigma_{\mathrm{e}}+2 k_{\mathrm{e}} \mathrm{F} \tilde{e}_{\mathrm{PG}}+k_{E E} \mathrm{~F}^{2} \tilde{e}_{\mathrm{PG}}^{2} .
$$

\section{Identification of the constitutive functions}

The general form of the diffusion coefficients is now restricted. Indeed, these coefficients are constrained by the equivalence of the two forms of the dissipation inequality shown in Sect. 5.1, by the compatibility conditions (5.20),(5.21), and further by the condition of positive (semi)definiteness of the generalized diffusion matrices. In addition, as pointed out in the Remark 3 above, experimental conditions that are used to measure the parameters should be specified : the analysis in Sects. 6.1 to 6.2 below considers that the ionic mobilities are measured at vanishing pore pressure gradient.

It is instrumental to introduce the following (pseudo-)vectors $\mathbf{N}$ and $\mathbf{Z}$ of length equal to the number $n_{\text {ion }}$ of ions:

$$
N_{k}=\frac{1}{\widehat{v}_{k}} \frac{n^{k E}}{n^{w E}}, \quad Z_{k}=\zeta_{k}, \quad k \in E^{\mathrm{ions}}
$$

Repeated use of electroneutrality will be made in the forms

$$
\mathbf{Z} \cdot \mathbf{Z}^{\perp}=0, \quad \mathbf{Z} \cdot \mathbf{N}=\mathbf{Z} \cdot \mathbf{N}_{\mathrm{nm}}=-\tilde{e}_{\mathrm{PG}} \cdot
$$

Here $\mathbf{Z}^{\perp}$ is the vector space of dimension $n_{\text {ion }}-1$ which is orthogonal to $\mathbf{Z}$. When mobile and non mobile ions are distinguished, $\mathbf{Z}^{\perp}$ may also assume the value $\mathbf{N}_{\text {mo }}$.

\subsection{A general form of diffusion with mobile and non mobile ions}

The format of the generalized diffusion equations is based on three key assumptions.

(D1) The hydraulic conductivity $K_{h}$ is known :

Measurement of hydraulic conductivity, at uniform concentrations of ions and PG's, and at vanishing electrical current $\mathbf{I}_{\mathrm{e} E}$, necessarily gives rise to a streaming potential $\nabla \phi_{E}=$ 
$-k_{\mathrm{e}} / \sigma_{\mathrm{e}} \mathbf{F}_{E}$. Thus $\mathbf{J}_{w E}=-k_{D} \mathbf{F}_{E}$, and if $k_{\mathrm{e}}>0$, water flows against pore pressure gradient but along the streaming potential gradient. The entity measured is thus the "open circuit" coefficient $k_{D}=k_{E E}-k_{\mathrm{e}}^{2} / \sigma_{\mathrm{e}}\left[\right.$ unit: $\left.\mathrm{m}^{3} \times \mathrm{sec} / \mathrm{kg}\right]$.

Alternatively, measurement of the flow at given fluid pressure, at uniform concentrations of ions and PG's, and uniform electrical potential yields the "short circuit" permeability $k_{E E}=\kappa_{w w}$, which is proportional to the hydraulic conductivity $K_{h}$, namely $\mathbf{J}_{w E}=$ $-k_{E E} \mathbf{F}_{E}$.

(D2) The effective ionic mobilities $u_{k}^{*}, k \in E^{\text {ions }}$, are known:

The velocity relative to water that the ionic species $k$ can reach under an electrical potential $\phi_{E}$ at uniform ionic concentrations and vanishing pore pressure gradient is $-u_{k}^{*} \operatorname{sgn} \zeta_{k} \nabla \phi_{E}$. The effective ionic mobility refers to the mobility in the porous structure, while the ionic mobility refers to a blank solution. The difference is introduced via the notion of tortuosity, described in Sect.6.3. The sign of the electrical charge indicates that a cation is moving towards the cathode, while an anion is moving towards the anode, i.e. in the direction of increasing electrical potential. In suspension mechanics and environmental geomechanics, this phenomenon known as electro-phoresis is used to densify fine particle suspensions around the anode; densified materials are removed periodically to allow for the process of anion elimination to continue. In agreement with the usual convention, the electrical current density has a direction opposite to that of electrons.

Since the diffusive flux involves both mobile and non mobile parts, there is an ambiguity on the volume fraction involved in the resulting flux, and, temporarily, the volume fraction is left undecided as $\tilde{n}^{k E}$, that is, at uniform ionic concentrations, $\mathbf{J}_{k E}^{d}=-\tilde{n}^{k E} u_{k}^{*} \operatorname{sgn} \zeta_{k} \nabla \phi_{E}$. The coefficients $k_{k \mathrm{e}}^{d}, k \in E^{\text {ions }}$, result as indicated in $(6.7)_{2}$. Then, using the definition of $k_{k \mathrm{e}}^{d}$ in (5.19), the coefficients $\kappa_{k l}^{d}$ are obtained to within a vector $\mathbf{A}$,

$$
\frac{\kappa_{k l}^{d}}{\widehat{v}_{k} \widehat{v}_{l}}=n^{E} \tilde{c}_{k E} \frac{u_{k}^{*}}{F\left|\zeta_{k}\right|} I_{k l}+A_{k} Z_{l}^{\perp} \quad k, l \in E^{\mathrm{ions}} .
$$

(D3) The matrix $\boldsymbol{\kappa}$ is enforced to be symmetric:

Upon insertion of the relation (6.3) in the definition (5.18), symmetry of

$$
\frac{\kappa_{k l}}{\widehat{v}_{k} \widehat{v}_{l}}=n^{E} \tilde{c}_{k E} \frac{u_{k}^{*}}{F\left|\zeta_{k}\right|} I_{k l}+A_{k} Z_{l}^{\perp}+N_{k} \frac{\kappa_{w l}}{\widehat{v}_{l}}, \quad k, l \in E^{\mathrm{ions}}
$$

implies

$$
A_{k} Z_{l}^{\perp}-Z_{k}^{\perp} A_{l}=\frac{\kappa_{w k}}{\widehat{v}_{k}} N_{l}-N_{k} \frac{\kappa_{w l}}{\widehat{v}_{l}}, \quad k, l \in E^{\mathrm{ions}} .
$$

A general solution of (6.5) involves three arbitrary coefficients $\alpha_{i}, i \in[1,3]$, and implies a particular structure of the $\kappa_{w k} / \widehat{v}_{k}$ 's, $k \in E^{\text {ions }}$,

$$
A_{k}=\alpha_{1} Z_{k}^{\perp}-\alpha_{2} N_{k}, \quad \frac{\kappa_{w k}}{\widehat{v}_{k}}=\alpha_{2} Z_{k}^{\perp}+\alpha_{3} N_{k}, \quad k \in E^{\text {ions }} .
$$

Hence, with help of (5.19), (6.4)-(6.6), the electrical conductivity and electro-osmotic coefficient are defined up to the coefficient $\alpha_{3}$. Moreover (6.6) implies $k_{E k}^{d} / \widehat{v}_{k}=\kappa_{k w}^{d} / \widehat{v}_{k}=$ $\kappa_{w k} / \widehat{v}_{k}-N_{k} \kappa_{w w}, k \in E^{\text {ions }}$, and, then $k_{k l}^{d} / \widehat{v}_{k} \widehat{v}_{l}=\kappa_{k l}^{d} / \widehat{v}_{k} \widehat{v}_{l}-N_{l} \kappa_{k w}^{d} / \widehat{v}_{k}, k, l \in E^{\text {ions }}$, results from (6.3). 
In summary,

$$
\left\{\begin{aligned}
k_{E E} & =\frac{K_{h}}{\rho_{w} \mathrm{~g}}>0, \quad k_{D}=k_{E E}-\frac{k_{\mathrm{e}}^{2}}{\sigma_{\mathrm{e}}} \\
\frac{k_{k \mathrm{e}}^{d}}{\widehat{v}_{k}} & =n^{E} \tilde{c}_{k E} u_{k}^{*} \operatorname{sgn} \zeta_{k}, k \in E^{\mathrm{ions}}, \\
\frac{k_{E k}^{d}}{\widehat{v}_{k}} & =\alpha_{2} Z_{k}^{\perp}+\left(\alpha_{3}-k_{E E}\right) N_{k}, k \in E^{\mathrm{ions}}, \\
\frac{k_{k l}^{d}}{\widehat{v}_{k} \widehat{v}_{l}} & =\alpha_{1} Z_{k}^{\perp} Z_{l}^{\perp}-\alpha_{2}\left(Z_{k}^{\perp} N_{l}+N_{k} Z_{l}^{\perp}\right)+\left(k_{E E}-\alpha_{3}\right) N_{k} N_{l} \\
& +n^{E} \tilde{c}_{k E} \frac{u_{k}^{*}}{\mathrm{~F}\left|\zeta_{k}\right|} I_{k l}, \quad k, l \in E^{\mathrm{ions}}, \\
k_{\mathrm{e}} & =-\alpha_{3} \mathrm{~F} \tilde{e}_{\mathrm{PG}}, \quad \sigma_{\mathrm{e}}=n^{E} \mathrm{~F} \sum_{k \in E^{\mathrm{ions}}}\left|\zeta_{k}\right| \tilde{c}_{k E} u_{k}^{*}+\alpha_{3} \mathrm{~F}^{2} \tilde{e}_{\mathrm{PG}}^{2}
\end{aligned}\right.
$$

The generalized diffusion matrix is left with three arbitrary coefficients $\alpha_{i}, i=[1,3]$. The relations (6.7) $)_{-7}$ agree with the compatibility condition (5.21). A formal way to define these coefficients is proposed in Sect.6.2.

The $4 \times 4$ diffusion matrix $\boldsymbol{\kappa}$ is known as soon as the $5 \times 5$ diffusion matrix $\mathcal{K}$ is, using successively $\kappa_{w w}=k_{E E},(6.6)$, and (6.4):

$$
\left\{\begin{array}{l}
\frac{\kappa_{w k}}{\widehat{v}_{k}}=\alpha_{2} Z_{k}^{\perp}+\alpha_{3} N_{k}, \quad k \in E^{\text {ions }} \\
\frac{\kappa_{k l}}{\widehat{v}_{k} \widehat{v}_{l}}=n^{E} \tilde{c}_{k E} \frac{u_{k}^{*}}{F\left|\zeta_{k}\right|} I_{k l}+\alpha_{1} Z_{k}^{\perp} Z_{l}^{\perp}+\alpha_{3} N_{k} N_{l}, \quad k, l \in E^{\text {ions }} .
\end{array}\right.
$$

In the above analysis, a single vector $\mathbf{Z}^{\perp}$ has been used. This covers completely the case of a binary electrolyte. For ternary electrolytes, two independent vectors $\mathbf{Z}^{\perp}$ should appear in e.g. (6.3).

To summarize the analysis at this point, let us emphasize that the only assumption of symmetry of the diffusion matrices and their algebraic equivalence has provided the general structure of the constitutive equations to within three scalars and $n_{\text {ion }}-1$ vectors of size $n_{\text {ion }}$.

\subsection{The particular case of arrowhead diffusion matrices}

A particular form of diffusion is derived below under the following assumptions:

- Assumption $(\mathcal{D} 1)$ : the diffusive flux of ions is not affected by a gradient of fluid pressure: thus $\alpha_{2}=0$ and $\alpha_{3}=k_{E E}$. In view of the symmetry of the diffusion matrix $\mathcal{K}$, the above assumption is equivalent to the fact that the ionic gradients do not affect the water flux;

- Assumption $(\mathcal{D} 2)$ : a gradient of concentration of ion $k$ does not affect the diffusive flux of ion $l \neq k$. Then $\alpha_{1}=0$. 
Therefore the diffusion matrix takes the symmetric arrowhead form

$$
\mathcal{K}=\left[\begin{array}{ccccc}
k_{E E} & 0 & 0 & 0 & k_{\mathrm{e}} \\
0 & k_{\mathrm{NaNa}}^{d} & 0 & 0 & k_{\mathrm{Nae}}^{d} \\
0 & 0 & k_{\mathrm{CaCa}}^{d} & 0 & k_{\mathrm{Cae}}^{d} \\
0 & 0 & 0 & k_{\mathrm{ClCl}}^{d} & k_{\mathrm{Cle}}^{d} \\
k_{\mathrm{e}} & k_{\mathrm{eNa}}^{d} & k_{\mathrm{eCa}}^{d} & k_{\mathrm{eCl}}^{d} & \sigma_{\mathrm{e}}
\end{array}\right],
$$

and its coefficients are given in terms of the hydraulic conductivity $K_{h}$, effective ionic mobilities $u_{k}^{*}$ and fixed charge $\tilde{e}_{\mathrm{PG}}$ as

$$
\left\{\begin{array}{l}
k_{E E}=\frac{K_{h}}{\rho_{w} \mathrm{~g}}>0, \quad k_{D}=k_{E E}-\frac{k_{\mathrm{e}}^{2}}{\sigma_{\mathrm{e}}} \geq 0, \\
\frac{k_{k \mathrm{e}}^{d}}{\widehat{v}_{k}}=n^{E} \tilde{c}_{k E} u_{k}^{*} \operatorname{sgn} \zeta_{k}, k \in E^{\mathrm{ions}} \\
\frac{k_{k l}^{d}}{\widehat{v}_{k} \widehat{v}_{l}}=n^{E} \tilde{c}_{k E} \frac{u_{k}^{*}}{\mathrm{~F}\left|\zeta_{k}\right|} I_{k l}, k, l \in E^{\mathrm{ions}}, \\
k_{\mathrm{e}}=-k_{E E} \mathrm{~F} \tilde{e}_{\mathrm{PG}}, \\
\sigma_{\mathrm{e}}=\sigma_{\mathrm{e}}^{\mathrm{ion}}+\sigma_{\mathrm{e}}^{\mathrm{PG}}, \quad \sigma_{\mathrm{e}}^{\text {ion }}=n^{E} \mathrm{~F} \sum_{k \in E^{\mathrm{ions}}}\left|\zeta_{k}\right| \tilde{c}_{k E} u_{k}^{*}, \quad \sigma_{\mathrm{e}}^{\mathrm{PG}}=k_{E E} \mathrm{~F}^{2} \tilde{e}_{\mathrm{PG}}^{2} \cdot
\end{array}\right.
$$

The short circuit permeability $k_{E E}$ is naturally positive. The open circuit permeability $k_{D}$ is positive as well due to the relation,

$$
\frac{k_{D}}{k_{E E}}=\frac{\sigma_{\mathrm{e}}^{\text {ion }}}{\sigma_{\mathrm{e}}} \geq 0
$$

Given the matrix $\mathcal{K}$, the matrix $\boldsymbol{\kappa}$ becomes

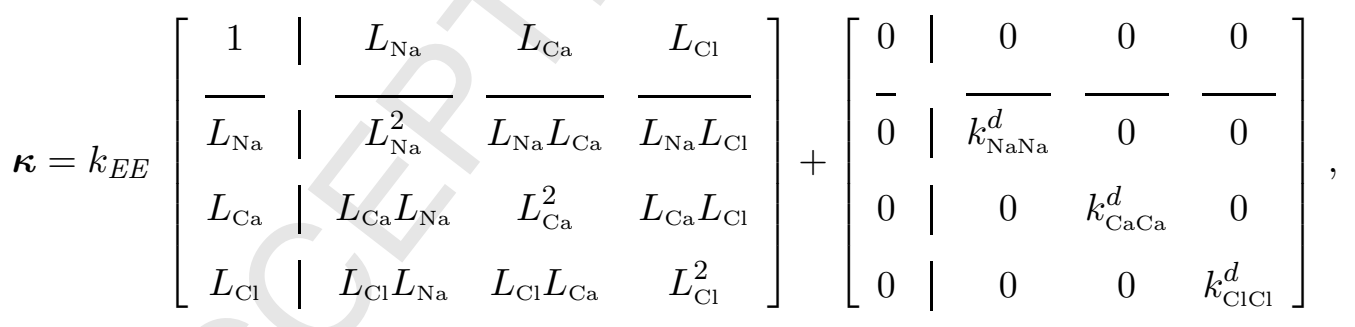

the $L^{\prime} s$ being defined as $L_{k}=n^{k E} / n^{w E}, k \in E^{\text {ions }}$. This formulation turns to be similar to that obtained by $\mathrm{Gu}$ et al. (1998) through another path. Gu et al. (1998) postulate the diffusion law in a format inverse to that used here. Indeed they write the gradients of electrochemical potentials in terms of fluxes through a frictional matrix, their eqn 14. They deduce the coefficients of the inverse relation that gives fluxes as functions of electrochemical potentials, their eqn 19, and finally the coefficients of the present generalized diffusion matrix $\mathcal{K}$. One should note however that the similarity of the generalized diffusion equations of the present approach and of $\mathrm{Gu}$ et al. (1998) is only formal because the repartition of species in phases is quite different.

A key assumption in $\mathrm{Gu}$ et al. (1998) is the fact that the frictional submatrix affected to ions is diagonal. This assumption can be shown to be equivalent to the submatrix $\kappa_{k l},(k, l) \in E^{\text {ions }}$ 
to be equal to a diagonal matrix plus a certain dyadic product: this implies the diffusive submatrix $k_{k l}^{d},(k, l) \in E^{\text {ions }}$ to be diagonal, which is indeed our Assumption $(\mathcal{D} 2)$. An algebraic proof is deferred to Appendix B.

\section{Remark 4: Back to the dissipation inequality}

Note that the diffusion matrix $\mathcal{K}$, eqn (6.9), is positive semi-definite because four of its principal minors $k_{E E}, k_{k k}^{d}, k \in E^{\text {ions }}$, are strictly positive while the matrix itself is singular. Therefore the dissipation $(3.1)_{3}$ due to diffusion is indeed positive or zero.

\section{Remark 5: Back to the identification procedures}

Since now the coefficients $k_{E k}^{d}, k \in E^{\text {ions }}$, are zero, then the identification procedures at vanishing gradient of pore pressure, and at vanishing flux of extrafibrillar water are identical, as observed by comparing eqns (5.16) and (5.17).

\subsection{Refinements and ranges of the coefficients}

The constitutive equations above introduce several parameters or functions. Typical values, gathered from literature, for human articular cartilage, Maroudas (1968), and for bovine cartilages, Mansour and Mow (1976), Frank and Grodzinsky (1987), Sachs and Grodzinsky (1989), Gu et al. (1998), Mow and Guo (2002), are listed below :

- $K_{h} \in\left[10^{-12}, 70 \times 10^{-12}\right.$ ] [unit: $\mathrm{m} / \mathrm{sec}$ ] is the hydraulic conductivity. In geomechanics, it is sometimes considered as varying with the void ratio according to the Kozeny-Carman formula, namely $K_{h} / K_{h 0}=\left(e / e_{0}\right)^{3}\left(1+e_{0}\right) /(1+e)$, where the subscript 0 indicates the reference (unstrained) state. As for articular cartilage, the data of Mansour and Mow (1976) indicate that the permeability decreases when increasing compressive strain while the bath pressure is fixed. Lai et al. (1981) consider relations of the form $K_{h} / K_{h 0}=$ $(1+e)^{M} /\left(1+e_{0}\right)^{M}$, or $K_{h} / K_{h 0}=\exp (M \operatorname{tr} \boldsymbol{\epsilon})$ : in the latter relation, $M$ is a positive parameter that may range up to 20. Holmes and Mow (1990) propose a two-parameter expression, namely, $K_{h} / K_{h 0}=\left(e / e_{0}\right)^{M_{1}} \exp \left(M_{2} \operatorname{tr} \boldsymbol{\epsilon}\right)$ with $M_{1} \sim 0.1$ and $M_{2} \sim 4-5$. In a finite deformation extension of this formula, Ateshian et al. (1997) take, for bovine articular cartilage as well, $M_{1}=2$ and $M_{2}=0.4-3.2$. Gu et al. (2004) use $M_{2}=0$ and $M_{1}$ around 3 for uncharged agarose gels and around 7 for porcine lumbar annulus fibrosus.

Indeed, the elastic modulus of cartilage is presumably, and actually, in direct relation with the collagen and GAG content. The hydraulic conductivity is on the other hand in inverse relation with GAG content, but independent of the ionic content, Maroudas (1975)(1979). Thus, as water is expelled during compression, the relative content of GAG increases and therefore the hydraulic conductivity is expected to decrease. In any case, the wide range of values of the parameters $M$ above indicate that the actual dependence of hydraulic conductivity in terms of strain is not completely resolved by these expressions, for at least two reasons. First, an assessment of these relations revolves around the definition of the void ratio! In the present three-phase context, the void ratio involved would be $e^{*}=$ $V_{E} /\left(V_{I}+V_{S}\right)$, while the void ratio $e$ in biphasic theories is $\left(V_{E}+V_{I}\right) / V_{S}$. Second, the actual permeability depends not only on the actual porosity, but also on the microstructure of this porosity. The notion of hydraulic tortuosity is briefly discussed below in relation with ionic mobilities and diffusion coefficients. 
Moreover, these relations imply that the mechanical and transport properties should vary across depth of the cartilage layer, as the chemical composition of cartilage does, Chen et al. (2001);

- $D_{k} \in\left[2 \times 10^{-10}, 20 \times 10^{-10}\right]\left[\right.$ unit: $\left.\mathrm{m}^{2} / \mathrm{sec}\right]$ is the coefficient of diffusion of the ion $k$;

- $u_{k} \in\left[8 \times 10^{-9}, 80 \times 10^{-9}\right]$ [unit: $\mathrm{m}^{2} / \mathrm{sec} /$ Volt] is the mobility of the ion $k$ in the porous medium. The ionic mobility is linked to the coefficient of diffusion by the Nernst-Einstein relation : $u_{k}=D_{k}\left|\zeta_{k}\right| \mathrm{F} / R T$.

The effective diffusion coefficient accounts for several aspects that imply diffusion in the porous medium to be slower than diffusion in a free infinitely dilute solution. For any ion, the coefficients of intrinsic diffusion $D$ and of effective diffusion $D^{*}=\tau D$ are linked by the tortuosity factor $\tau<1$ (this entity is sometimes defined as the inverse of the one used here). A similar relation applies to effective ionic mobilities, namely $u^{*}=\tau u$. One contribution to the tortuosity factor $\tau$ stems from geometrical considerations, namely the actual path that an ion has to travel in a porous medium is larger than in a blank solution. For a cross-linked polymer membrane, the tortuosity factor $\tau$ for ions that do not interact with the membrane has been defined, using statistical arguments, by Mackie and Meares (1955), as a function of the relative volumes of water and solid, namely $\tau=\tau_{1}(e)=e^{2} /(2+e)^{2}$ for a two-phase mixture. This relation would take the format $\tau=\tau_{1}\left(e^{*}\right)$ in the present three-phase context. Mechanical compression expels first extrafibrillar water out of the cartilage, and therefore, according to this model, it reduces considerably the tortuosity factor and therefore the effective diffusion coefficients. A formula of the same flavor which goes by the name of Archie's law is used for rocks by the geophysical community, where the tortuosity factor is postulated as a power relationship $n^{m-1}$, where $n$ is a (model-dependent) fluid volume fraction. The exponent $m$ varies in the typical range $[1.5,2.5]$ for cemented, or not cemented, granular materials. It reaches the minimum value 1.5 for a random array of spheres, and it is greater than this value for flat particles.

As such, the above formulas have a main drawback: the tortuosity factor $\tau_{1}$ accounts solely for geometrical aspects of the pore structure. However, the presence of fixed charges modifies considerably the effective coefficients of diffusion and ionic mobilities. Indeed, at small concentrations, the range of action of the fixed charge is large, and the ionic mobilities are reduced. This aspect may be inserted in the tortuosity factor $\tau$ which can be viewed as the product $\tau_{1} \tau_{2}$ of two terms, the Mackie-Meares's geometrical factor $\tau_{1}$, and a second factor of electro-chemical nature $\tau_{2}$ that vanishes at infinitely small concentrations of mobile ions. It is customary to adopt

Assumption $(\mathcal{T})$ : tortuosity affects all ions identically;

- $\sigma_{\mathrm{e}} \in\left[10^{-2}, 1\right][$ unit: Siemens/m=A/Volt/m] is the bulk electrical conductivity of the porous medium. Notice that the electrical conductivity given in the format (6.10) is not constant, it assumes a minimal non zero value for distilled water and it increases with ionic strength. The various constituents of cartilage contribute to the electrical conductivity:

(1) In the present model, the collagen network is considered infinitely resistive. The intrafibrillar ions are not involved either. However, mass transfers and the trend toward chemical equilibrium between the intra- and extrafibrillar phases affect indirectly the extrafibrillar ionic concentrations and volume of water, and therefore the tortuosity factor. 
So, the possibility that increasing high extrafibrillar ionic strength be accompanied by a decrease of electrical conductivity can not be ruled out a priori.

(2) The contribution of the PG's is strictly positive, except at the isoelectric point where the fixed charge vanishes. The isoelectric $\mathrm{pH}$ is reported to be around 2.75 by Frank and Grodzinsky (1987)-I. Note that, if PG's were part of the solid phase, they would not enter in $\sigma_{\mathrm{e}}$ in (6.10), nor would the non mobile ions, Loret et al. (2004).

(3) Ions in the extrafibrillar fluid phase contribute additively, with an intensity proportional to the product of their effective ionic mobilities and of their concentrations. Here, the mobilities are taken constant. However, according to Kohlrausch's square root law, they decrease with concentration $c$, say generically as $u=u_{0}-\left(\alpha u_{0}+\beta\right) \sqrt{c}$, where $u_{0}$ is the electrical conductivity at dilute concentration, $\alpha$ and $\beta$ are positive parameters. Therefore, the contribution of an ion to the overall conductivity, essentially proportional to $c u^{*}$, would not increase linearly with concentration, but would tend to flatten, or possibly to decrease at higher concentration.

(4) Non-mobile extrafibrillar ions can be made to either contribute or not, depending whether, in (6.10), $\tilde{n}^{k E}$ is set to $n^{k E}$ or to $n_{\mathrm{mo}}^{k E}$. Only the former option is pursued in the sequel.

- $k_{\mathrm{e}} \in\left[0.1 \times 10^{-8}, 5 \times 10^{-8}\right.$ ] [unit: $\mathrm{m}^{2} / \mathrm{sec} /$ Volt] is the electro-osmotic coefficient. According to the Helmholtz-Smoluchowski theory, it should be proportional to the fixed charge, with opposite sign. The expression $(6.10)_{4}$ is in perfect agreement with these requirements. As $\mathrm{pH}$ decreases, the charge of PG's becomes less negative. It vanishes at the isoelectric point, and it becomes positive at lower pH, Grodzinsky et al. (1981). Therefore, in absence of pressure and concentration gradients, a difference of electrical potential induces an osmotic water flow towards the cathode at neutral and high $\mathrm{pH}$, and towards the anode at low $\mathrm{pH}$, a phenomenon termed "reverse osmosis" not to be confused with "anomalous negative osmosis" investigated below.

The general trends of electrical conductivity and of some other indicators of hydraulic and electrical transport are displayed on Figs. 3 and 4. Some features are worth of notice:

- for the reference $\mathrm{CEC}=43 \mathrm{kC} / \mathrm{kg}$, the ionic conductivity tends to vary linearly with the ionic strength of the bath $c_{\mathrm{Cl} B}$ for $c_{\mathrm{Cl} B} \geq 0.2 \mathrm{M}$. The influence of the fluid volume fraction would be moderated if the tortuosity would follow Archie's law;

- as CEC decreases to zero, the electrical conductivity tends to $\mathrm{F} n^{E} c_{\mathrm{Cl} B}\left(u_{k}^{*}+u_{\mathrm{Cl}}^{*}\right)$;

- the difference in electrical conductivity between the sodium chloride and calcium chloride electrolytes is small;

- as expected, the relative contribution of ions to the electrical conductivity increases when the ionic strength of the bath increases, when the fluid volume fraction increases, and when the CEC decreases. The influence of the fluid volume fraction would be amplified if the tortuosity would follow Archie's law;

- the ratio of open and short circuit permeabilities may be significatively smaller than 1 at low ionic strength;

- the ratio $k_{\mathrm{e}} / \sigma_{\mathrm{e}}$ decreases as the ionic strength of the bath increases, but varies non monotonously in terms of fluid volume fraction and CEC. For articular cartilages under physiological conditions, the ratio takes values around $1 \times 10^{-8} \mathrm{Volt} / \mathrm{Pa}$, in agreement with measurements by Frank and Grodzinsky (1987)II and Huyghe et al. (2002). 
The trends may be checked against data available in the literature, where both the fixed charge density and fluid volume fractions are known accurately. For example, Huyghe et al. (2002) analyzed a hydrogel with fixed charge of -0.15 mole per liter of fluid tissue, with fluid volume fraction of 0.977 , in contact with a bath at $0.15 \mathrm{M}$ of $\mathrm{NaCl}$. They report the following measurements: $k_{\mathrm{e}}=0.88 \times 10^{-8} \mathrm{~m}^{2} / \mathrm{sec} /$ Volt and $\sigma_{\mathrm{e}}=0.76$ Siemens $/ \mathrm{m}$, so that the ratio $k_{\mathrm{e}} / \sigma_{\mathrm{e}}$ is equal to $1.16 \times 10^{-8} \mathrm{Volt} / \mathrm{Pa}$. These data yield the short circuit permeability $k_{E E}=-k_{\mathrm{e}} / \mathrm{F} / e_{\mathrm{PG}}=0.61 \times 10^{-15} \mathrm{~m}^{4} / \mathrm{N} / \mathrm{sec}$ and the open circuit permeability $k_{D}=k_{E E}-k_{\mathrm{e}}^{2} / \sigma_{\mathrm{e}}=0.51 \times 10^{-15} \mathrm{~m}^{4} / \mathrm{N} / \mathrm{sec}$, so that $k_{D} / k_{E E}=0.83$. Assuming $\rho_{\text {dry }}=1800 \mathrm{~kg} / \mathrm{m}^{3}$, the corresponding CEC is $347.4 \mathrm{kC} / \mathrm{kg}$ : this value is much larger than in articular cartilages due to the high fluid volume fraction. The model yields values quite in the range of experimental measurements, namely $k_{D} / k_{E E}=0.82, \sigma_{\mathrm{e}}=1.15 \mathrm{Siemens} / \mathrm{m}$, $k_{\mathrm{e}} / \sigma_{\mathrm{e}}=1.54 \times 10^{-8}$ Volt $/ \mathrm{Pa}$.

While the model of generalized diffusion does not introduce explicitly an osmotic coefficient, the latter is in fact hidden in the equations, as shown in the next section.

\section{The reflection, or osmotic, coefficient}

Let a cartilage specimen to be in contact with a bath. Its mechanical and chemical state is in general spatially non uniform. Still, each material point of the cartilage can be viewed to be in equilibrium with a fictitious bath. Of course, if the cartilage state is spatially uniform, then the local fictitious baths become all identical to the real bath. This is the situation we have in mind below when we will plot the reflection coefficient and the diffusion coefficients as a function of the chemical composition of a bath.

The two compartments, namely the extrafibrillar phase, and the real or fictitious baths, are in chemical equilibrium, they contain the same species, and they both satisfy electroneutrality. The fixed charge, assumed to be spatially uniform, is given by $(2.4)_{2}$.

Assuming the chemical composition of the fictitious or real baths to be given, the composition of the extrafibrillar phase is deduced first, Sect.7.1. Two particular electrolytes are envisaged, namely a binary symmetric electrolyte $\mathrm{NaCl}$, and a binary nonsymmetric electrolyte $\mathrm{CaCl}_{2}$, denoted by the index $k=\mathrm{Na}$ and $k=\mathrm{Ca}$ respectively.

On comparing the ionic fluxes in the cartilage and in the bath, Sect.7.3. a reflection coefficient is evidenced as characterizing the filtration properties of the fixed charged medium. Indeed, at small ionic concentrations of the bath, and at small ionic concentrations of the mobile ions in the cartilage, the range of influence of the fixed charge is large, and $\omega$ is close to one. Thus the co-ions, and, by electroneutrality, the counter-ions, are repelled. As the ionic concentrations increase, the range of influence of the fixed charge decreases, leaving ions more freedom to diffuse, and the reflection coefficient tends to vanish.

Another interpretation of the reflection coefficient can be proposed, Sect. 7.5. Indeed, the fluid fluxes in the cartilage and in the bath can be compared, rather than the ionic fluxes. This comparison features the osmotic coefficient. Osmotic and reflection coefficients are shown to be one and the same.

\subsection{Equilibrium relations for the two binary electrolytes}

In view of the electroneutrality of each of the two compartments,

$$
x_{\mathrm{Cl} B}=\zeta_{k} x_{k B}, \quad x_{\mathrm{Cl} E}=\zeta_{k} x_{k E}+y_{\mathrm{PG}}, \quad y_{\mathrm{PG}} \equiv \zeta_{\mathrm{PG}} x_{\mathrm{PG}},
$$


the chemical potentials of the salts $\mathrm{NaCl}$ or $\mathrm{CaCl}_{2}$ can be brought in the form (2.12). The mechanical contribution to the chemical potential of the salts is now considered small with respect to the chemical contributions. Then equilibrium of the salt between these two compartments implies

$$
x_{k E}\left(x_{\mathrm{Cl} E}\right)^{\zeta_{k}}=x_{k B}\left(x_{\mathrm{Cl} B}\right)^{\zeta_{k}}, \quad k=\mathrm{Na}, \mathrm{Ca} .
$$

The variations of the molar fractions of the cations are connected by the relation

$$
\frac{d x_{k E}}{d x_{k B}}=\frac{d x_{\mathrm{Cl} E}}{d x_{\mathrm{Cl} B}}=\frac{1+\zeta_{k}}{x_{k B}} \frac{x_{k E} x_{\mathrm{Cl} E}}{\zeta_{k}^{2} x_{k E}+x_{\mathrm{Cl} E}}, \quad k=\mathrm{Na}, \mathrm{Ca} .
$$

The equilibrium of the chemical potentials of water between the two compartments provides the osmotic pressure $p_{w E}-p_{B}$ (neglecting the concentration $c_{\mathrm{PG}}$ of PG's) as

$$
\frac{\widehat{v}_{w}}{R T}\left(p_{w E}-p_{B}\right) \simeq y_{\mathrm{PG}}+\left(1+\zeta_{k}\right)\left(x_{k E}-x_{k B}\right), \quad k=\mathrm{Na}, \mathrm{Ca} .
$$

The explicit relations between the molar fractions in the two compartments are now detailed.

For the $\mathrm{NaCl}$ electrolyte, the extrafibrillar molar fractions express in terms of the molar fractions of the bath as

$$
x_{\mathrm{NaE}}=-\frac{y_{\mathrm{PG}}}{2}+\sqrt{\left(\frac{y_{\mathrm{PG}}}{2}\right)^{2}+x_{\mathrm{NaB}}^{2}}, \quad x_{\mathrm{ClE}}=\frac{y_{\mathrm{PG}}}{2}+\sqrt{\left(\frac{y_{\mathrm{PG}}}{2}\right)^{2}+x_{\mathrm{NaB}}^{2}} .
$$

For the $\mathrm{CaCl}_{2}$ electrolyte, the molar fraction of cations calcium is obtained via the positive solution $y=\sqrt{x_{\mathrm{CaE}}}$ of the cubic equation,

$$
y^{3}+\frac{y_{\mathrm{PG}}}{2} y-\left(\sqrt{x_{\mathrm{Ca} B}}\right)^{3}=0, \quad x_{\mathrm{CaE}}=y^{2}, \quad x_{\mathrm{ClE}}=2 y^{2}+y_{\mathrm{PG}} .
$$

Conversely, for both binary electrolytes, the ionic molar fractions in the bath express in terms of the ionic molar fractions of the extrafibrillar phase as

$$
x_{k B}=\frac{x_{\mathrm{ClB}}}{\zeta_{k}}=\left(x_{k E}\left(\frac{x_{\mathrm{ClE}}}{\zeta_{k}}\right)^{\zeta_{k}}\right)^{\left(1+\zeta_{k}\right)^{-1}} .
$$

\subsection{The absolute fluxes for an open circuit}

For open electrical circuit, $\mathbf{I}_{\mathrm{e} E}=\mathbf{0}$, the electrical field $\mathbf{F}_{\mathrm{e} E}$ can be expressed in terms of the gradients of fluid pressure $\mathbf{F}_{E}$ and ionic concentrations $\mathbf{F}_{l E}^{d}$, namely

$$
\mathbf{F}_{\mathrm{e} E}=-\frac{k_{\mathrm{e}}}{\sigma_{\mathrm{e}}} \mathbf{F}_{E}-\frac{1}{\sigma_{\mathrm{e}}} \sum_{l \in E^{\text {ions }}} \frac{k_{\mathrm{e} l}^{d}}{\widehat{v}_{l}} \widehat{v}_{l} \mathbf{F}_{l E}^{d},
$$

Then, using the constitutive equations (5.11),(6.9), the flux of water becomes

$$
\mathbf{J}_{w E}=-k_{D} \mathbf{F}_{E}+\frac{k_{\mathrm{e}}}{\sigma_{\mathrm{e}}} \sum_{l \in E^{\text {ions }}} \frac{k_{\mathrm{e} l}^{d}}{\widehat{v}_{l}} \widehat{v}_{l} \mathbf{F}_{l E}^{d},
$$

while, with (A.1), the ionic fluxes relative to the solid $\mathbf{J}_{k E}$ can be recast in the format

$$
\frac{\mathbf{J}_{k E}}{\widehat{v}_{k}}=-k_{k E}^{0} \mathbf{F}_{E}-\sum_{l \in E^{\text {ions }}} k_{k l E}^{0} \widehat{v}_{l} \mathbf{F}_{l E}^{d},
$$

with coefficients :

$$
\left\{\begin{array}{l}
\widehat{v}_{k} k_{k E}^{0}=-\frac{k_{\mathrm{e}}}{\sigma_{\mathrm{e}}} k_{k \mathrm{e}}^{d}+k_{D} \frac{n^{k E}}{n^{w E}} \\
\widehat{v}_{k} \widehat{v}_{l} k_{k l E}^{0}=k_{k l}^{d}-\frac{k_{k \mathrm{e}}^{d} k_{\mathrm{el}}^{d}}{\sigma_{\mathrm{e}}}-\frac{k_{\mathrm{e}}}{\sigma_{\mathrm{e}}} \frac{n^{k E}}{n^{w E}} k_{\mathrm{el}}^{d}, \quad l \in E^{\mathrm{ions}} .
\end{array}\right.
$$




\subsection{The reflection coefficient as a filtration indicator}

First let us note that an experiment with open circuit, involving in addition uniform concentrations of ions and PG's, can be checked to be feasible. Moreover, the pressure gradient $\mathbf{F}_{E}$ generates a fluid flux $\mathbf{J}_{w E}=-k_{D} \mathbf{F}_{E}$.

Since the electrical current density vanishes in the extrafibrillar phase, eqn (2.19), then, the fluxes of water (7.9) and ions (7.10) satisfy the relations:

$$
\zeta_{k} \frac{\mathbf{J}_{k E}}{\widehat{v}_{k}}=\frac{\mathbf{J}_{\mathrm{Cl} E}}{\widehat{v}_{\mathrm{Cl}}}=\zeta_{k} \frac{k_{k E}^{0}}{k_{D}} \mathbf{J}_{w E}=\frac{k_{\mathrm{Cl} E}^{0}}{k_{D}} \mathbf{J}_{w E} .
$$

Similarly in the fictitious bath,

$$
\zeta_{k} \frac{\mathbf{J}_{k B}}{\widehat{v}_{k}}=\frac{\mathbf{J}_{\mathrm{Cl} B}}{\widehat{v}_{\mathrm{Cl}}}=\zeta_{k} \frac{c_{k B}}{n^{w B}} \mathbf{J}_{w B}=\frac{c_{\mathrm{Cl} B}}{n^{w B}} \mathbf{J}_{w B}
$$

In a one directional flow context, the reflection coefficient is defined as the relative difference between the cationic or anionic fluxes in the extrafibrillar and bath compartments, namely $\mathbf{J}_{k E}=(1-\omega) \mathbf{J}_{k B}$ and $\mathbf{J}_{\mathrm{Cl} E}=(1-\omega) \mathbf{J}_{\mathrm{Cl} B}$, at identical fluid fluxes in the bath and cartilage, $\mathbf{J}_{w B}=\mathbf{J}_{w E}$. Using (6.10) and (7.11)-(7.13), the reflection coefficient $\omega$ results as

$$
\omega=1-\frac{c_{\mathrm{Cl} E}}{c_{\mathrm{Cl} B}} \frac{\zeta_{k} c_{k E}\left(u_{k}+u_{\mathrm{Cl}}\right)}{\zeta_{k} c_{k E} u_{k}+c_{\mathrm{Cl} E} u_{\mathrm{Cl}}}, \quad k=\mathrm{Na}, \mathrm{Ca} .
$$

Assumption $(\mathcal{T})$ has been used to eliminate the tortuosity factor, as well as the fact that $c_{w B}$ and $c_{w E}$ are quite close.

To interpret this coefficient, we view the bath composition as given, and deduce the chemical content of the cartilage as indicated by (7.5), or (7.6). As the cationic, or anionic, concentration in the bath increases from zero, the extrafibrillar chloride content varies much slower than in the bath, as indicated by (7.2), (7.3) and (7.7): thus the reflection coefficient for a fresh bath water is one, as expected.

For infinitesimal fixed charge, the bath and extrafibrillar concentrations are equal and $\zeta_{k} c_{k E}$ is equal to $c_{\mathrm{Cl} E}$. Then $\omega$ decreases quickly to 0 as soon as the ionic strength of the bath increases. The situation is similar when the ionic content tends to overweigh the fixed charge. Indeed, the filtration properties depend crucially of the fixed charge content.

Figure 5 shows the variations of the reflection coefficient as a function of the bath anionic strength, for the two electrolytes $\mathrm{NaCl}$ and $\mathrm{CaCl}_{2}$. The effects of the $\mathrm{CEC}$ and volume fraction of the fluid are highlighted. Some comments are in order :

- the reflection coefficient decreases quickly from 1 to 0 as the ionic strength of the bath increases;

- at given fluid volume fraction and at small ionic strength, the higher the CEC, the stronger the filtration effect of the fixed charge, and the higher the reflection coefficient. The trend is opposite at large ionic strength;

- at given CEC, the higher the fluid volume fraction, the lower the reflection coefficient at small ionic strength. Indeed, a large fluid volume fraction reduces the fixed charge density, and compacted materials are known to be more efficient ion screens. The trend is again opposite at large ionic strength;

- the calcium chloride electrolyte is endowed with a smaller reflection coefficient than the sodium chloride electrolyte.

These features agree with the data reported on $\mathrm{Ca}^{2+}$-soil system by Kemper and Quirk (1972). 


\subsection{Negative osmosis}

A negative "anomalous" value of the reflection coefficient is reported in the experimental data of Kemper and Quirk (1972) on kaolinites, bentonites and illites, and analyzed in Olsen et al. (1990) for soil systems, and in $\mathrm{Gu}$ et al. (1997) for articular cartilages.

In the above experimental conditions, $\mathbf{J}_{k E}=(1-\omega) \mathbf{J}_{k B}$. Consequently, a negative osmosis implies a larger ionic flux in the cartilage than in the bath.

Using (7.3) and (7.7), an extremum of $\omega$ in terms of the bath concentration is defined by

$$
c_{\mathrm{Cl} B}^{\min }=\frac{e_{\mathrm{PG}}}{\alpha-\beta}(\alpha(1-\beta))^{\beta}((1-\alpha) \beta)^{1-\beta}, \quad \omega^{\min }=1-\left(\frac{\beta}{\alpha}\right)^{\beta}\left(\frac{1-\beta}{1-\alpha}\right)^{1-\beta},
$$

where

$$
\alpha=\frac{u_{k}}{u_{k}+u_{\mathrm{Cl}}}, \quad \beta=\frac{\zeta_{k}}{\zeta_{k}+1} .
$$

Clearly, while the extremum concentration is proportional to the fixed charge density, the minimum reflection coefficient depends only on $\alpha$ and $\beta$, that is, on the relative ionic mobilities and on the valence of the counterion. It is encouraging to observe that the experimental curves obtained for $\mathrm{Ca}^{2+}$-soil systems by Kemper and Quirk (1972) and reported in Bresler (1973) and Mitchell (1993) show precisely the trends produced by the model and displayed on Fig. 5. Moreover, the experimental negative osmosis is more marked for illites and kaolinites whose fixed charge is much smaller than in montmorillonites: the experiments and simulations for bentonites in Leroy (2005) do not show negative osmosis. The experimental negative osmosis is more marked as well for $\mathrm{Ca}^{2+}$-soils than for $\mathrm{Na}^{+}$-soils. These two additional features are displayed by the model. The actual minimum reflection coefficient is -0.022 for the $\mathrm{NaCl}$ electrolyte and -0.111 for the $\mathrm{CaCl}_{2}$ electrolyte, irrespective of $\mathrm{CEC}$ and volume fraction. However, the experimental minima increase, in algebraic value, as the fixed charge increases. We may just speculate that calcium-binding to the fixed charge, a phenomenon not accounted for here, might be a reason for this increase of the minima.

For a negative fixed charge, the condition of existence of this extremum is $\alpha<\beta$, that is

$$
u_{\mathrm{Cl}}>\frac{u_{k}}{\zeta_{k}}
$$

This condition is verified for the two electrolytes of interest. The above results on negative osmosis complete and generalize the analysis of $\mathrm{Gu}$ et al. (1997) which addresses only a sodium chloride electrolyte.

The condition $\alpha<\beta$ ensures the minimum of $\omega$ to be negative, and thus, ensures the existence of a concentration $c_{\mathrm{Cl} B}^{0}$ at which $\omega$ vanishes, e.g. for the sodium chloride electrolyte,

$$
c_{\mathrm{Cl} B}^{0}=e_{\mathrm{PG}} \frac{\alpha(1-\alpha)}{2 \alpha-1},
$$

which blatantly is proportional to the fixed charge density and depends on the ratio of ionic mobilities. The algebraic expression for calcium chloride can be obtained as well as a solution of a quadratic equation.

It might be worth to recall that the curves shown on Fig. 5 can not be used directly to follow a chemical experimental where the bath composition is varied, since then the fluid volume fractions changes as well, due to chemo-mechanical coupling. Still, as the ionic strength of the bath is increased, the reflection coefficient will necessarily go through its minimum. 


\subsection{Another point of view: the diffusion-osmosis phenomenon}

Another derivation is proposed now, which features a coefficient that can be termed 'osmotic coefficient'.

The pressure gradient $\mathbf{F}_{E}$, which is essentially equal to $\nabla p_{w E}$, is obtained via (7.4), namely

$$
\mathbf{F}_{E}=\nabla p_{w E}=\nabla p_{B}+R T \nabla e_{\mathrm{PG}}+R T\left(1+\zeta_{k}\right)\left(\nabla c_{k E}-\nabla c_{k B}\right) .
$$

The fluid flux is considered to be driven by an osmotic gradient in the underlying baths, namely

$$
\mathbf{J}_{w E}=-k_{D}\left(\nabla p_{B}-\omega R T\left(1+\zeta_{k}\right) \nabla c_{k B}\right) .
$$

Assuming a uniform fluid pressure, $\nabla p_{B}=\mathbf{0}$, and a uniform distribution of PG's, $\nabla e_{\mathrm{PG}}=\mathbf{0}$, we recover the standard presentation of the osmotic coefficient, viewed as moderating the influence of a chemical gradient on the flow. The algebraic expression (7.14) is retrieved by equating the two expressions of the fluid flux, provided on one hand by (7.9) where $\mathbf{F}_{E}$ is given by (7.19), and on the other hand by (7.20), with help of (6.11) and (7.3). If $\omega$ is negative, relation (7.20) indicates that water flows against, and not along, the chemical gradient, which has led the phenomenon to be coined "anomalous osmosis".

Remark 6. The difference in electrical fields in actual setups

Experiments to explore the osmosis effect typically consist in maintaining the specimen between a lower bath and an upper bath, with identical pressures, but distinct concentrations. The difference between the upper and lower electrical fields is observed to change sign for an average concentration slightly larger than the one that marks negative osmosis. It would be worth to check this aspect by the model.

Remark 7. Fixed charge: in the solid or fluid phases?

As mentioned in the introduction, the definition of phases in articular cartilages is not unambiguous. The fixed charge is of primary concern here. According to a kinematic criterion, it would be part of the solid phase. On the other hand, its mechanical effects are related to its concentration with respect to the volume of extrafibrillar water. Some key differences between the two options are listed below:

1. Fixed charge in the solid phase.

This is the option taken in the modeling of expansive clays in Loret et al. (2004).

Let us first consider the chemo-mechanical equilibrium of a clay with an external reservoir: pore pressure, concentration and electrical potential are continuous at the boundary between the water phase and the reservoir. Moreover, the electrical conductivity vanishes when the external reservoir is quasi-distilled water.

In addition, the electro-osmotic coefficient $k_{\mathrm{e}}$ and the osmotic coefficient $\omega$ are not constrained by compatibility conditions. Thus they have to be provided by additional constitutive equations.

2. Fixed charge in the fluid phase.

The presence of the fixed charge in the extrafibrillar phase has two main consequences. First, pore pressure, concentrations and electrical potential undergo a jump across the interface that separates the cartilage and a bath, even at equilibrium. This observation is of importance in a finite element context. Indeed, they strongly guide the choice of the primary variables. 
Second, the electro-osmotic coefficient $k_{\mathrm{e}}$ and the osmotic coefficient $\omega$ are constrained by compatibility conditions : in fact, they are provided by the formulation, in explicit or implicit form.

\section{Experimental diffusion coefficients}

Typical experimental setups provide information on the diffusion coefficients: the results are naturally recorded as function of the chemical composition of the bath. The interpretation requires caution. Indeed, the presence of the proteoglycans in the extrafibrillar water implies the cartilage specimen to be surrounded by a fictitious membrane across which a number of entities are discontinuous. Now, the constitutive equations are phrased in terms of intra- or extrafibrillar entities. The experimental data should be re-plotted in terms of these entities to provide the intrinsic material functions.

\subsection{Open circuit and no net fluid flux}

Let us consider a cartilage layer with open electrical circuit, $\mathbf{I}_{\mathrm{e} E}=\mathbf{0}$, and no net fluid flux, $\mathbf{J}_{w E}=\mathbf{0}$. Then, with help of (6.11), the pressure gradient $\mathbf{F}_{E}$ and electrical field $\mathbf{F}_{\mathrm{e} E}$ can be calculated in terms of the concentration gradients $\mathbf{F}_{l E}^{d}$ as

$$
\mathbf{F}_{\mathrm{e} E}=-\frac{k_{E E}}{k_{\mathrm{e}}} \mathbf{F}_{E}=-\frac{1}{\sigma_{\mathrm{e}}^{\text {ion }}} \sum_{l \in E^{\text {ions }}} \frac{k_{\mathrm{e} l}^{d}}{\widehat{v}_{l}} \widehat{v}_{l} \mathbf{F}_{l E}^{d} .
$$

Upon backsubstitution, the diffusive fluxes become,

$$
-\frac{\mathbf{J}_{k E}^{d}}{\widehat{v}_{k}}=\sum_{l \in E^{\text {ions }}} k_{k l E}^{*} \widehat{v}_{l} \mathbf{F}_{l E}^{d},
$$

where

$$
k_{k l E}^{*}=\frac{k_{k l}^{d}}{\widehat{v}_{k} \widehat{v}_{l}}-\frac{1}{\sigma_{\mathrm{e}}^{\text {ion }}} \frac{k_{k \mathrm{e}}^{d}}{\widehat{v}_{k}} \frac{k_{\mathrm{el}}^{d}}{\widehat{v}_{l}}, \quad l \in E^{\mathrm{ions}} .
$$

The two binary electrolytes $\mathrm{NaCl}$ and $\mathrm{CaCl}_{2}$ are considered in turn.

Using (5.15) with $\mathbf{I}_{\mathrm{e} E}=\mathbf{0}$ and $\mathbf{J}_{w E}=\mathbf{0}$ implies the diffusive fluxes of the ion and cation to be parallel:

$$
\begin{aligned}
& -\zeta_{k} \frac{\mathbf{J}_{k E}^{d}}{\widehat{v}_{k}} \quad=\quad-\frac{\mathbf{J}_{\mathrm{Cl} E}^{d}}{\widehat{v}_{\mathrm{Cl}}}= \\
& =\zeta_{k} \sum_{l \in E^{\text {ions }}} k_{k l E}^{*} \widehat{v}_{l} \mathbf{F}_{l E}^{d}=\sum_{l \in E^{\text {ions }}} k_{\mathrm{Cl} l E}^{*} \widehat{v}_{l} \mathbf{F}_{l E}^{d} .
\end{aligned}
$$

Assuming a spatially uniform distribution of PG's, electroneutrality yields $\zeta_{k} \nabla x_{k E}=\nabla x_{\mathrm{Cl} E}$. Substituting this relation in (8.4), and using the definition (5.11) of the driving gradients $\mathbf{F}_{l E}^{d}$, $l=k, \mathrm{Cl}$, the diffusive fluxes [unit: $\mathrm{m}^{-2} \mathrm{sec}^{-1}$ ] of the cation, $k=\mathrm{Na}$ or $\mathrm{Ca}$, and of the chlorine ion can be cast in the format:

$$
-\zeta_{k} \frac{\mathbf{J}_{k E}^{d}}{\widehat{v}_{k}}=\zeta_{k} \bar{D}_{k} \nabla c_{k E}=-\frac{\mathbf{J}_{\mathrm{Cl} E}^{d}}{\widehat{v}_{\mathrm{Cl}}}=\bar{D}_{\mathrm{Cl}} \nabla c_{\mathrm{Cl} E}
$$

The diffusion coefficients [unit: $\mathrm{m}^{2} \mathrm{sec}^{-1}$ ] $\bar{D}_{k}$ and $\bar{D}_{\mathrm{Cl}}$ are equal,

$$
\bar{D}_{k}=R T\left(\frac{k_{k k E}^{*}}{c_{k E}}+\zeta_{k} \frac{k_{k \mathrm{Cl} E}^{*}}{c_{\mathrm{Cl} E}}\right)=\bar{D}_{\mathrm{Cl}}=R T\left(\frac{1}{\zeta_{k}} \frac{k_{\mathrm{Cl} k E}^{*}}{c_{k E}}+\frac{k_{\mathrm{Cl} \mathrm{l} E}^{*}}{c_{\mathrm{Cl} E}}\right),
$$


and denoted $\bar{D}$,

$$
\bar{D}=\frac{R T}{\mathrm{~F}} \frac{n^{E}}{\zeta_{k}} \frac{\left(\zeta_{k}^{2} c_{k E}+c_{\mathrm{Cl} E}\right) u_{\mathrm{Cl}}^{*} u_{k}^{*}}{\zeta_{k} c_{k E} u_{k}^{*}+c_{\mathrm{Cl} E} u_{\mathrm{Cl}}^{*}}, \quad k=\mathrm{Na}, \mathrm{Ca}
$$

The following limits hold:

- the limit value of the diffusion coefficient $\bar{D}$ at infinitely small ionic strength of the bath, the cartilage is in equilibrium with, is

$$
\lim _{c_{\mathrm{Cl} B}=\zeta_{k} c_{k B} \rightarrow 0} \bar{D}=\frac{R T}{\mathrm{~F}} n^{E} u_{\mathrm{Cl}}^{*}
$$

- the limit value of the diffusion coefficient $\bar{D}$ of a cartilage in absence of fixed charge $\left(y_{\mathrm{PG}}=0\right.$ or $e_{\mathrm{PG}}=0$ ) is independent of the ionic strength of the bath, and

$$
\lim _{y \mathrm{PG} \rightarrow 0} \bar{D}=\frac{R T}{\mathrm{~F}} n^{E} \frac{1+\zeta_{k}}{\zeta_{k}} \frac{u_{\mathrm{Cl}}^{*} u_{k}^{*}}{u_{\mathrm{Cl}}^{*}+u_{k}^{*}}, \quad \forall c_{\mathrm{Cl} B}=\zeta_{k} c_{k B}, \quad k=\mathrm{Na} \text { or } \mathrm{Ca} .
$$

\subsection{Application to a cartilage layer maintained between two baths}

As an application, a cartilage layer of thickness $h$ is placed between two baths of controlled chemical content: a slight concentration difference is imposed between the two baths. The experiment is realized at no fluid flow and open circuit. The diffusion coefficient associated to this experiment, denoted by $D_{B}^{\exp }\left[\right.$ unit: $\left.\mathrm{m}^{2} \mathrm{sec}^{-1}\right]$, is defined as ( $\nabla$ denotes the difference between the two baths divided by the thickness $h$ )

$$
\bar{D} \nabla c_{k E}=D_{B}^{\exp } \nabla c_{k B}, \quad k=\mathrm{Na} \text { or Ca } .
$$

Hence, with help of (7.3) and Assumption $(\mathcal{T})$,

$$
D_{B}^{\exp }=\frac{R T}{\mathrm{~F}} n^{E}\left(1+\zeta_{k}\right) \tau \frac{c_{\mathrm{Cl} E}}{c_{\mathrm{Cl} B}} \frac{c_{k E} u_{\mathrm{Cl}} u_{k}}{\zeta_{k} c_{k E} u_{k}+c_{\mathrm{Cl} E} u_{\mathrm{Cl}}}, \quad k=\mathrm{Na} \text { or Ca } .
$$

Figure 6 displays the effects of the ionic strength of the bath, of the fluid volume fraction and of the fixed charge density, on the experimental diffusion coefficient $D_{B}^{\exp }$ divided by the tortuosity factor $\tau$. The experimental coefficient $D_{B}^{\exp }$ can be observed to increase with the ionic strength of the baths, starting from a null value, and reaching a plateau at larger ionic strength, two features observed in the experiments of Malusis and Shackelford (2002) on a bentonite, a geological material with fixed charge. The latter plays a crucial role here: indeed in absence of fixed charge, the experimental diffusion coefficient $D_{B}^{\exp }$ assumes the constant value given by (8.9).

Further details may be worth of interest:

- for the values of ionic mobilities used, there always exists an ionic strength at which the experimental diffusion coefficient presents a maximum. The existence and position of this maximum have been investigated in relation with the osmotic coefficient;

- at given fluid volume fraction $n^{E}$, the higher the CEC, the smaller the experimental diffusion coefficient at small ionic strength; the trend is opposite at large ionic strength;

- at given CEC, the higher the fluid volume fraction, the higher the experimental diffusion coefficient;

- the $\mathrm{Ca}^{2+}$-electrolyte is endowed with an experimental diffusion coefficient that is slightly larger, at small ionic strength, than the monovalent electrolyte; the trend is opposite at large ionic strength. 
It is worthwhile to observe that the above experimental diffusion coefficient, eqn (8.11), can be expressed in terms of the transmission coefficient $1-\omega$, eqn (7.14), namely

$$
D_{B}^{\exp }=\frac{R T}{\mathrm{~F}} n^{E} \frac{1+\zeta_{k}}{\zeta_{k}} \frac{u_{k} u_{\mathrm{Cl}}}{u_{k}+u_{\mathrm{Cl}}} \tau(1-\omega), \quad k=\mathrm{Na} \text { or } \mathrm{Ca} .
$$

A relation of this type has been suggested in the geomechanical literature on the grounds that $D_{B}^{\exp }$ and $1-\omega$ both vanish at infinitesimal ionic strength of the baths. The present analysis shows that such a relation does not need to be postulated as a basic constitutive equation: indeed it results from the constitutive equations of diffusion.

\section{Concluding remarks}

The framework for this analysis of articular cartilages is purely macroscopic, in the sense that the actual details of the geometry and microstructure at the nanoscale are wiped out. On the other hand, the implicit averaging process has kept track of the features that are thought to govern the chemo-mechanical behavior, e.g.

- the presence of fixed charge on the proteoglycans which influences mechanics through electrical shielding, swelling and shrinking, and transport via the osmotic effect;

- the existence of two types of water, inside the collagen fibrils, and around the proteoglycans, of different chemical compositions. This feature endows both the mechanical and transport properties with instantaneous and delayed responses.

Indeed, even if the mechanical behaviors of the individual pieces are time independent (nonviscous), the overall behavior of the cartilage is indeed time-dependent and it displays several characteristic times, namely a time associated to seepage, times associated to the diffusion of ions, and times associated to mass transfers between the intra- and extrafibrillar compartments.

In Loret and Simões (2004)(2005)a, simulations of a succession of equilibria were performed in order to test the chemo-mechanical constitutive equations only, and mechanical and chemical equilibria were assumed to hold between the two water phases and between the extrafibrillar phase and the bath. The framework described here endows the material behavior with intrinsic times. It allows to simulate laboratory experiments with characteristic loading times which may be smaller, of the same order or larger than the various material time scales. The transient response depends strongly on the relative positions of these characteristic times. The issue is addressed and illustrated in a companion paper via the finite element method.

Note that the effect of $\mathrm{pH}$ on the electrical conductivity and electro-osmotic coefficient might be considered to be accounted for due to the explicit presence of the negative fixed charge. However, to be complete, the model should be enriched by constitutive equations that provide the evolution of this fixed charge as the $\mathrm{pH}$ of the surrounding, which is a priori different from the extrafibrillar $\mathrm{pH}$, varies. In fact, both the mechanical and transport properties of materials endowed with a fixed electric charge are strongly modified by alkaline and acid environments, e.g. Gajo and Loret (2007) for a brief review. Preliminary data indicate that $\mathrm{pH}$ changes affect substantially the diffusion coefficients of artificial joints, Kitano et al. (2001).

Acknowledgements - The work of F.S. was financed by Fundação para a Ciência e a Tecnologia (FCT) through the project POCTI/ECM/46759/2002 "Electro-chemo-mechanical couplings in soils and biological porous media". 


\section{References}

Ateshian G., Warden W.H., Kim J.J., Grelsamer R.P. and Mow V.C. (1997). Finite deformation biphasic material properties of bovine articular cartilage from confined compression experiments. J. Biomechanics, 30(11-12), 1157-1164.

Basser P.J., Schneiderman R., Bank R.A., Wachtel E. and Maroudas A. (1998). Mechanical properties of the collagen network in human articular cartilage as measured by osmotic stress technique. Archives Biochemistry Biophysics, 351(2), 207-219.

Bresler, E. (1973). Anion exclusion and coupling effects in nonsteady transport through unsaturated soils. I. Theory. Soil Science Society of America J., 37, 663-669.

Chen A.C., Bae W.C., Shinagl R.M. and Sah R.L. (2001). Depth- and strain-dependent mechanical and electromechanical properties of full-thickness bovine articular cartilage in confined compression. $J$. Biomechanics, 34, 1-12.

Eisenberg S.R. and Grodzinsky A.J. (1985). Swelling of articular cartilage and other connective tissues: electromechanical forces. J. Orthopaedic Research, 3, 148-159.

Frank E.H. and Grodzinsky A.J. (1987). Cartilage electromechanics, J. Biomechanics, 20(6), I. Electrokinetic transduction and the effects of electrolyte $\mathrm{pH}$ and ionic strength, 615-627. II. A continuum model of cartilage electrokinetics and correlation with experiments, 629-639.

Gajo A. and Loret B. (2007). The mechanics of active clays circulated by salts, acids and bases. J. of the Mechanics and Physics of Solids, accepted for publication.

Grodzinsky A.J., Roth V., Myers E., Grossman W.D. and Mow V.C. (1981). The significance of electromechanical and osmotic forces in the nonequilibrium swelling behavior of articular cartilage in tension. J. Biomech. Engng., Transactions of the ASME, 103, 221-231.

Gu W.Y., Lai W.M. and Mow V.C. (1997). A triphasic analysis of negative osmotic flows through charged hydrated soft tissues. J. Biomechanics, 30(1), 71-78.

Gu W.Y., Lai W.M. and Mow V.C. (1998). A mixture theory for charged hydrated soft tissues containing multi-electrolytes: passive transport and swelling behaviors. J. Biomech. Engng., Transactions of the ASME, 120, 169-180.

Gu W.Y., Yao H., Vega A.L. and Flager D. (2004). Diffusivity of ions in agarose gels and intervertebral discs: effect of porosity. Annals Biomedical Engng., 32(12), 1710-1717.

Holmes M.H and Mow V.C. (1990). The non-linear characteristics of soft gels and hydrated connective tissues in ultrafiltration. J. Biomechanics, 23, 1145-1156.

Huyghe J. (1999). Intra-extrafibrillar mixture formulation of soft charged hydrated tissues. J. Theoretical and Applied Mechanics, 3, 37, 519-536.

Huyghe J. and Janssen J.D. (1999). Thermo-chemo-electro-mechanical formulation of saturated charged porous solids. Transport in Porous Media, 34, 129-141.

Huyghe J.M., Janssen C.F., Lanir Y., van Donkelaar C.C., Maroudas A. and van Campen D. (2002). Experimental measurement of electrical conductivity and electro-osmotic permeability of ionised porous media. in Porous media: theoretical, experimental and numerical applications, W. Ehlers and J. Bluhm eds., 295-313, Springer Verlag, Berlin.

Huyghe J.M., Houben G.B., Drost M.R. and C.C. van Donkelaar (2003). An ionised/non-ionised dual porosity model of intervertebral disc tissue. Biomechanics and Modeling in Mechanobiology, 2(1), 3-19.

Kemper W.D. and Quirk J.P. (1972). Ionic mobilities and electric charges of external clay surfaces inferred from potential differences and osmotic flow. Soil Science Society of America J., 36, 426-433.

Kitano T., Ateshian G.A., Mow V.C., Kadoya Y. and Yamano Y. (2001). Constituents and pH changes in protein rich hyaluronan solution affect the biotribological properties of artificial articular joints. $J$. Biomechanics, 34, 1031-1037.

Lai W.M., Mow V.C. and Roth V. (1981). Effects of non-linear stress-strain dependent permeability and rate of compression on the stress behavior of articular cartilage. J. Biomech. Engng., Transactions 
of the ASME, 103, 61-66.

Lai W.M., Hou J.S. and Mow V.C. (1991). A triphasic theory for the swelling and deformation behaviors of articular cartilage. J. Biomech. Engng., Transactions of the ASME, 113, 245-258.

Leroy Ph. (2005). Transport ionique dans les argiles. Influence de la microstructure et des effects d'interface. PhD thesis, Université Aix-Marseille III.

Li S.-T. and Katz E.P. (1976). An electrostatic model for collagen fibrils. The interaction of reconstituted collagen with $\mathrm{Ca}^{++}, \mathrm{Na}^{+}$, and $\mathrm{Cl}^{-}$. Biopolymers, 15, 1439-1460.

Loix F., Simões F.M.F. and Loret B. (2006). Articular cartilage with intra- and extrafibrillar watersSimulations of transient salt replacements by the finite element method. In preparation.

Loret B., Gajo A. and Simões F.M.F. (2004). A note on the dissipation due to generalized diffusion with electro-chemo-mechanical couplings in heteroionic clays. Eur. J. Mechanics-A/Solids, 23(5), 763-782.

Loret B. and Simões F.M.F. (2004). Articular cartilage with intra- and extrafibrillar waters. A chemomechanical model. Mechanics of Materials, 36(5-6), 515-541.

Loret B. and Simões F.M.F. (2005)a. Mechanical effects of ionic replacements in articular cartilage. Biomechanics and Modeling in Mechanobiology, 4(2-3). Part I- The constitutive model, 63-80; Part IISimulations of successive substitutions of $\mathrm{NaCl}$ and $\mathrm{CaCl}_{2}, 81-99$.

Loret B. and Simões F.M.F. (2005)b. A framework for deformation, generalized diffusion, mass transfer and growth in multi-species multi-phase biological tissues, Eur. J. Mechanics-A/Solids, 24(5), 757-781.

Mackie J.S. and Meares P. (1955). The diffusion of electrolytes in a cation-exchange resin membrane-I. Proc. R. Soc. Lond., 232, 498-509.

Malusis M.A. and Shackelford C.D. (2002). Coupling effects during steady-state solute diffusion through a semipermeable clay membrane. Environmental Science and Technology, 36, 1312-1319.

Mansour J.M. and Mow V.C. (1976). The permeability of articular cartilage under compressive strain and high pressures. J. Bone and Joint Surgery, 58A, 509-516.

Maroudas A. (1968). Physico-chemical properties of cartilage in the light of ion exchange theory. Biophysical J., 8, 575-595.

Maroudas, A. (1975) Biophysical chemistry of cartilaginous tissues with special reference to solute and fluid transport. Biorheology, 12, 233-248.

Maroudas A. (1979). Physico-chemical properties of articular cartilage. In Adult articular cartilage, M.A.R. Freeman ed., Tunbridge Wells, England, 215-290.

Maroudas A., Wachtel E., Grushko G., Katz E. P. and Weinberg P. (1991). The effect of osmotic and mechanical pressures on water partitioning in articular cartilage. Biochimica et Biophysica Acta, 1073, 285-294.

Mitchell, J.K. (1993). Fundamentals of Soil Behavior. 2nd ed., J. Wiley \& Sons, Chichester.

Mow V.C. and Guo X.E. (2002). Mechano-electrochemical properties of articular cartilage: their inhomogeneities and anisotropies. Annual Review Biomedical Engng., 4, 175-209.

Olsen H.W., Yearsley E.N. and Nelson K.R. (1990) Chemico-osmosis versus diffusion-osmosis. Transportation Research Record - Soils, Geology and Foundations, Washington DC, 1288, 15-22.

Sachs J.R. and Grodzinsky A.J. (1989). An electromechanically coupled poroelastic medium driven by an applied electric current: surface detection of bulk material properties. Physico-Chemical Hydrodynamics, 11(4), 585-614.

Torzilli P.A. (1985). Influence of cartilage conformation on its equilibrium water partition. J. Orthopaedic Research, 3, 473-483.

Werner A. and Gründer W. (1999). Calcium-induced structural changes of cartilage proteoglycans studied by ${ }^{1} \mathrm{H}$ NMR relaxometry and diffusion measurements. Magnetic Resonance in Medicine, 341, 43-50. 


\section{Appendix A: Relations between fluxes}

Notice the relations between the fluxes defined in Sect. 2.4.1,

$$
\mathbf{J}_{k E}=\mathbf{J}_{k E}^{d}+n^{k E}\left(\mathbf{v}_{w E}-\mathbf{v}_{S}\right)=\mathbf{J}_{k E}^{d}+\frac{n^{k E}}{n^{w E}} \mathbf{J}_{w E}, \quad k \in E^{\mathrm{mo}} .
$$

Let

$$
n_{*}^{E} \equiv \sum_{k \in E^{\mathrm{mo}}} n^{k E} \neq n_{\mathrm{mo}}^{E} \equiv \sum_{k \in E^{\mathrm{mo}}} n_{\mathrm{mo}}^{k E}
$$

and

Then

$$
\rho_{*}^{E} \equiv \sum_{k \in E^{\mathrm{mo}}} n^{k E} \rho_{k} \neq \rho_{\mathrm{mo}}^{E} \equiv \sum_{k \in E^{\mathrm{mo}}} n_{\mathrm{mo}}^{k E} \rho_{k}
$$

and

as well as

$$
\mathbf{J}_{E}=\sum_{k \in E^{\mathrm{mo}}} \mathbf{J}_{k E}^{d}+\frac{n_{*}^{E}}{n^{w E}} \mathbf{J}_{w E}=\sum_{k \in E} \mathbf{J}_{k E}^{d}+\frac{n^{E}}{n^{w E}} \mathbf{J}_{w E},
$$

$$
\frac{\mathbf{J}_{w E}}{n^{w E}}=\frac{1}{n^{k E}}\left(\mathbf{J}_{k E}-\mathbf{J}_{k E}^{d}\right)=\frac{1}{n_{*}^{E}} \sum_{l \in E^{\mathrm{mo}}} \mathbf{J}_{l E}-\mathbf{J}_{l E}^{d}, \quad k \in E^{(\mathrm{mo})},
$$

$$
\sum_{k \in E^{\mathrm{mo}}} \mathbf{M}_{k E}=\sum_{k \in E^{\mathrm{mo}}} \rho_{k} \mathbf{J}_{k E}=\frac{\rho_{*}^{E}}{n_{*}^{E}} \mathbf{J}_{E}+\sum_{k \in E^{\mathrm{mo}}}\left(\rho_{k}-\frac{\rho_{*}^{E}}{n_{*}^{E}}\right) \mathbf{J}_{k E}^{d} .
$$

\section{Appendix B: Structure of diffusion matrices}

The relations (5.1),(5.2) link the fluxes, relative to the solid, of water $\mathbf{J}$ and ions $\mathbf{J}_{i}, i \in[1,3]$, to the gradients of the electrochemical potentials of the associated species $\mathbf{M}$ and ions $\mathbf{M}_{\mathbf{i}}, i \in[1,3]$. They may be rewritten in the simplified format (the matrix below plays the role of the matrix $\boldsymbol{\kappa}$ in (6.12)):

$$
\left[\begin{array}{c}
\mathbf{J} \\
\mathbf{J}_{1} \\
\mathbf{J}_{2} \\
\mathbf{J}_{3}
\end{array}\right]=\left[\begin{array}{cccc}
1 & n_{1} & n_{2} & n_{3} \\
n_{1} & c_{11} & c_{12} & c_{13} \\
n_{2} & c_{21} & c_{22} & c_{23} \\
n_{3} & c_{31} & c_{32} & c_{33}
\end{array}\right]\left[\begin{array}{c}
\mathbf{M} \\
\mathbf{M}_{1} \\
\mathbf{M}_{2} \\
\mathbf{M}_{3}
\end{array}\right]
$$

The electrochemical potentials may be expressed in terms of the diffusive fluxes as

$$
\left[\begin{array}{l}
\mathbf{M}_{1} \\
\mathbf{M}_{2} \\
\mathbf{M}_{3}
\end{array}\right]=\mathbf{B}\left[\begin{array}{l}
\mathbf{J}_{1}-n_{1} \mathbf{J} \\
\mathbf{J}_{2}-n_{2} \mathbf{J} \\
\mathbf{J}_{3}-n_{3} \mathbf{J}
\end{array}\right], \quad \mathbf{M}=\mathbf{J}-\sum_{j=1,3} n_{j} \mathbf{M}_{j}
$$

With $\mathbf{C}$ the matrix of components $c_{i j},(i, j) \in[1,3]^{2}$, the matrix $\mathbf{B}$ above is defined as

$$
\mathbf{B}=(\mathbf{C}-\mathbf{n} \otimes \mathbf{n})^{-1} .
$$

The matrix form of the complete inverse relation is

$$
\left[\begin{array}{c}
\mathbf{M} \\
\mathbf{M}_{1} \\
\mathbf{M}_{2} \\
\mathbf{M}_{3}
\end{array}\right]=\left[\begin{array}{cccc}
1-\sum_{j, k} B_{j k} & -\sum_{j} B_{j 1} & -\sum_{j} B_{j 2} & -\sum_{j} B_{j 3} \\
-\sum_{j} B_{1 j} & B_{11} & B_{12} & B_{13} \\
-\sum_{j} B_{2 j} & B_{21} & B_{22} & B_{23} \\
-\sum_{j} B_{3 j} & B_{31} & B_{32} & B_{33}
\end{array}\right]\left[\begin{array}{c}
\mathbf{J} \\
\mathbf{J}_{1} \\
\mathbf{J}_{2} \\
\mathbf{J}_{3}
\end{array}\right] .
$$

The above matrix is proportional to the matrix $\mathbf{A}$ in $\mathrm{Gu}$ et al. (1998), their eqn 17. They require the lower major to be diagonal. This restriction implies the inverse of our matrix $\mathbf{C}-\mathbf{n} \otimes \mathbf{n}$, and thus $\mathbf{C}-\mathbf{n} \otimes \mathbf{n}$, to be diagonal. Equivalently our matrix $\mathbf{C}$ has to be the sum of a diagonal part, plus a dyadic part, as indeed can be checked on (6.12). Now, the matrix $\mathbf{C}$ represents the submatrix of $\boldsymbol{\kappa}$ given by $(6.8)_{2}$, and the matrix $\mathbf{C}-\mathbf{n} \otimes \mathbf{n}$ represents the central part of the diffusive matrix noted $k_{k l}^{d}, k, l \in E^{\text {ions }}$, and given by $(6.7)_{5}$ for $\alpha_{1}=\alpha_{2}=0, \alpha_{3}=k_{E E}$. 


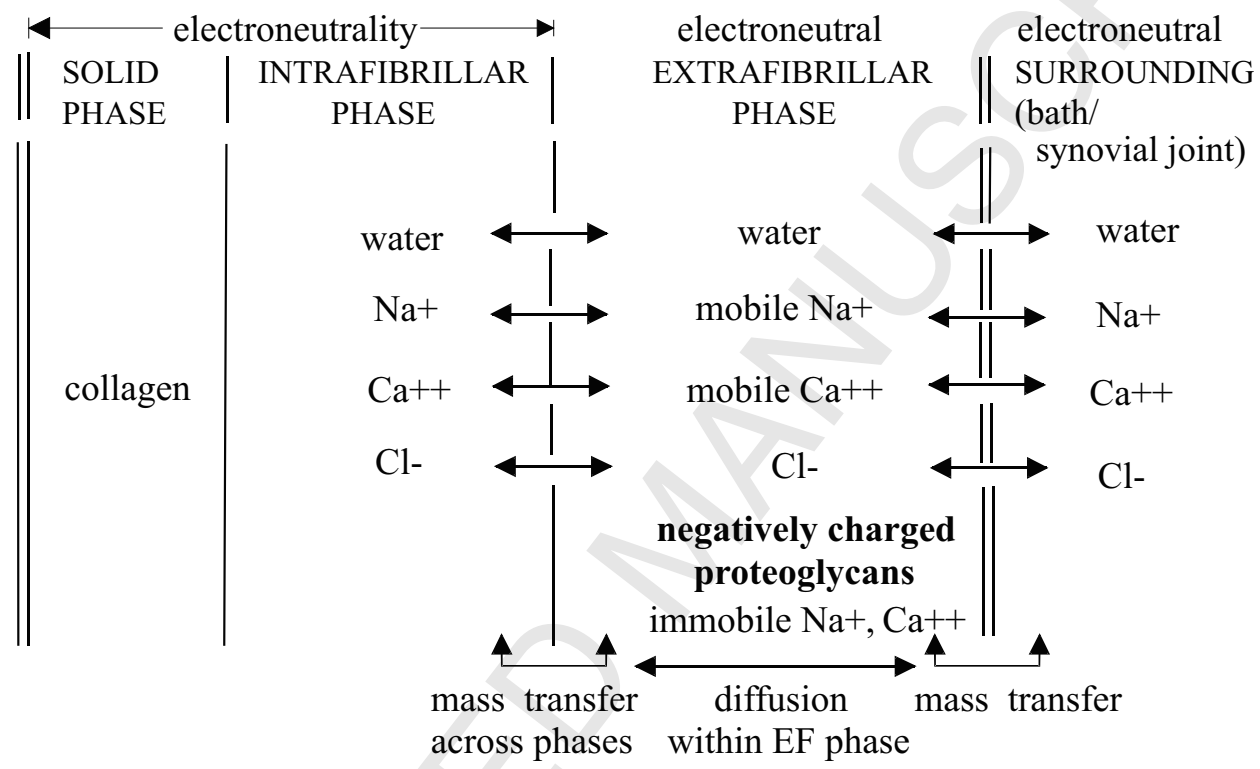

Figure 1 Articular cartilage is partitioned in three phases, one solid phase and two fluid phases, Loret and Simões (2005)a. Each fluid phase contains several species. Some of these species are mobile, at least partially: water and ions can enter and leave the intrafibrillar space defined by collagen fibrils. Proteoglycans which are macromolecules are too large to be admitted into that space. Water and ions can also be exchanged between the extrafibrillar phase and the exterior. 


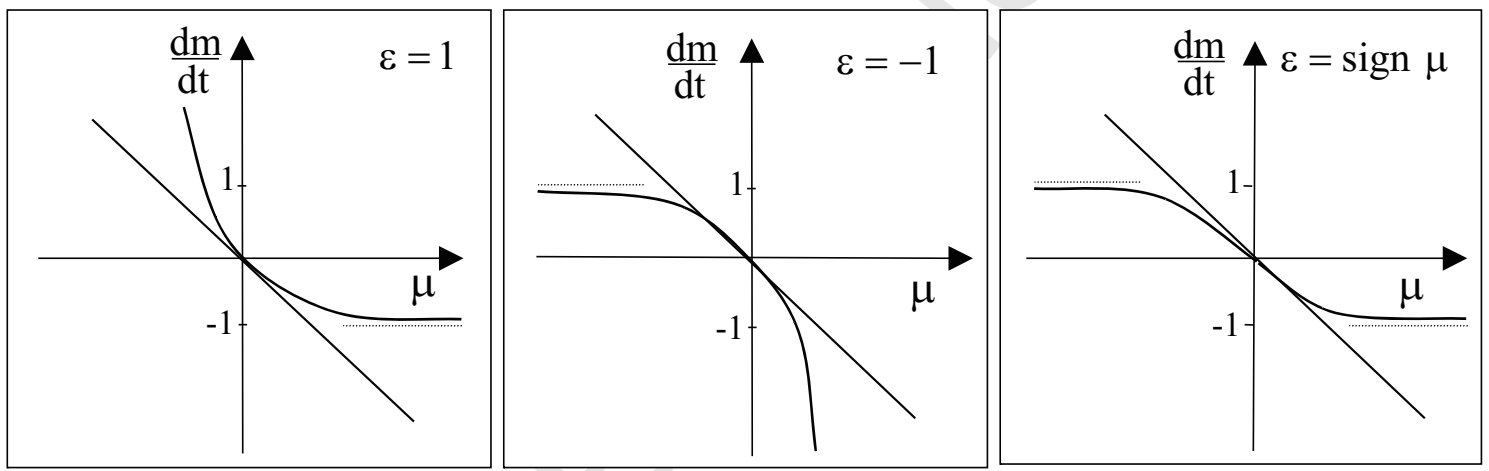

Figure 2 Exponential transfer laws $d m / d t=(\exp (-\epsilon A \mu)-1) \epsilon$, with $A>0$, behave differently depending on the sign of $\epsilon$, but, close to equilibrium, they are tangent to the linear law $d m / d t=-A \mu$. 

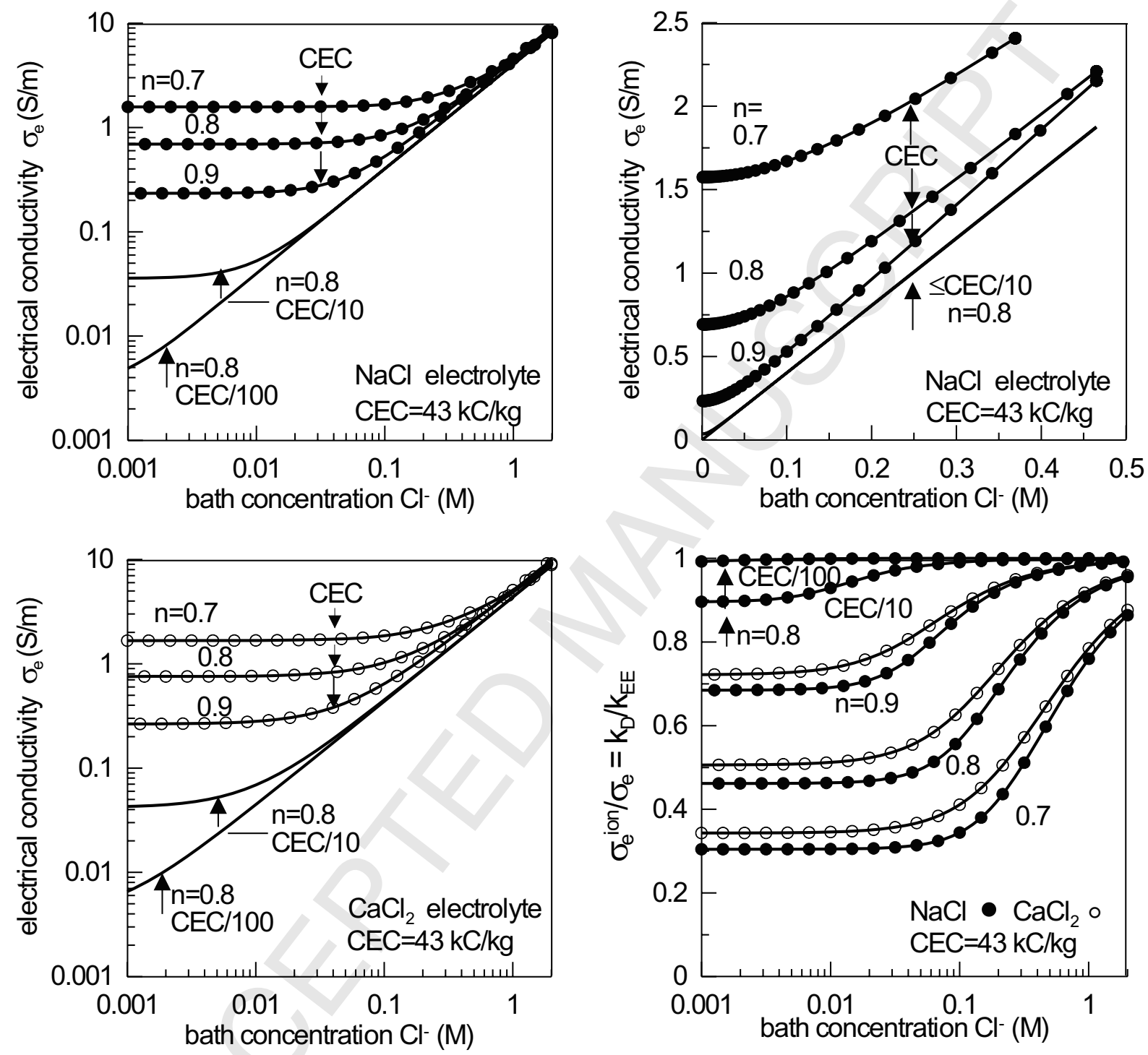

Figure 3 Effects of ionic strength of the bath, of fluid volume fraction $n$ and of CEC on the electrical conductivity of articular cartilages.

The parameters used are as follows: ionic mobilities [unit $: 10^{-9} \mathrm{~m}^{2} / \mathrm{sec} /$ Volt] $: u_{\mathrm{Na}}=51.75$, $u_{\mathrm{Ca}}=61.75, u_{\mathrm{Cl}}=79.0$; constant tortuosity factor $=0.4$; constant hydraulic permeability $k_{E E}=$ $10^{-15} \mathrm{~m}^{3} \times \mathrm{sec} / \mathrm{kg}$, and fluid volume fractions and CEC as indicated on the curves. 



Figure 4 Effects of ionic strength of the bath, of fluid volume fraction $n$ and of CEC on the coefficient of streaming potential of articular cartilages. Same parameters as in Fig. 3.
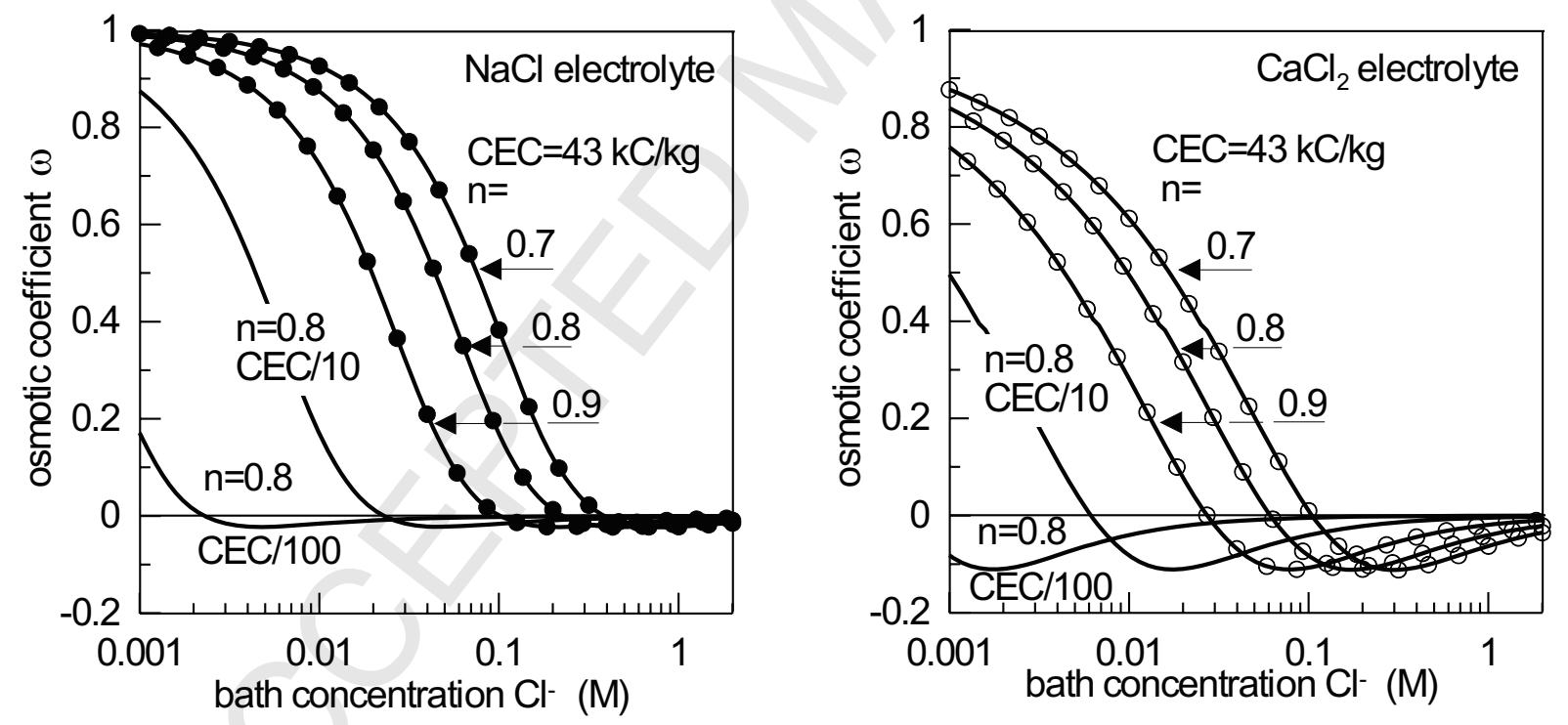

Figure 5 Reflection, or osmotic, coefficient $\omega$ for articular cartilages in contact with a bath containing either a sodium chloride electrolyte or a calcium chloride electrolyte. The parameters used are: ionic mobilities as defined on Fig. 3, dry density $\rho_{\text {dry }}=1800 \mathrm{~kg} / \mathrm{m}^{3}$, fluid volume fraction $n$ and CEC as indicated on the curves.

For the values of ionic mobilities used, there always exists an ionic strength given by (7.18) above which the osmotic coefficient is negative. The negative minimum value of the osmotic coefficient is defined by (7.15). 

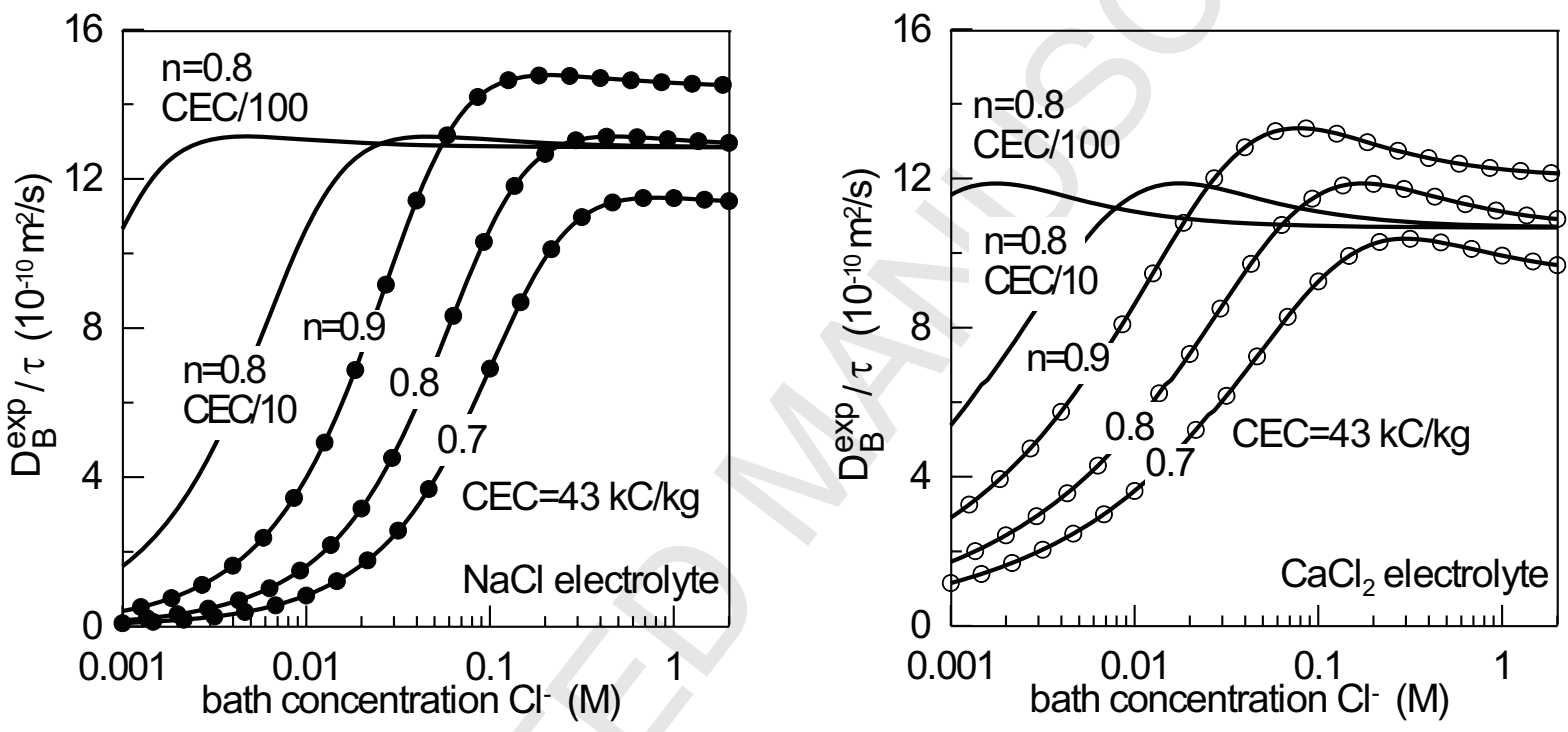

Figure 6 Experimental diffusion coefficient $D_{B}^{\exp }$ divided by the tortuosity factor $\tau$ as a function of the ionic strength of the bath. Same parameters as on Fig. 5 . 